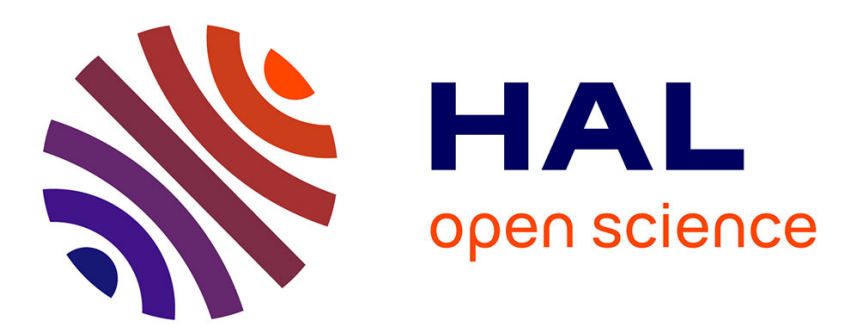

\title{
Dynamics of the first single Grandjean-Cano line in cholesterics under weak applied magnetic fields
}

\author{
G. Malet, J. Marignan, O. Parodi
}

\section{To cite this version:}

G. Malet, J. Marignan, O. Parodi. Dynamics of the first single Grandjean-Cano line in cholesterics under weak applied magnetic fields. Journal de Physique, 1976, 37 (7-8), pp.865-882. 10.1051/jphys:01976003707-8086500 . jpa-00208482

\section{HAL Id: jpa-00208482 https://hal.science/jpa-00208482}

Submitted on 1 Jan 1976

HAL is a multi-disciplinary open access archive for the deposit and dissemination of scientific research documents, whether they are published or not. The documents may come from teaching and research institutions in France or abroad, or from public or private research centers.
L'archive ouverte pluridisciplinaire HAL, est destinée au dépôt et à la diffusion de documents scientifiques de niveau recherche, publiés ou non, émanant des établissements d'enseignement et de recherche français ou étrangers, des laboratoires publics ou privés. 


\title{
DYNAMICS OF THE FIRST SINGLE GRANDJEAN-CANO LINE IN CHOLESTERICS UNDER WEAK APPLIED MAGNETIC FIELDS
}

\author{
G. MALET, J. MARIGNAN and O. PARODI
}

Groupe de dynamique des phases condensées (*), Laboratoire de cristallographie, Université des sciences et techniques du Languedoc, place Eugène-Bataillon, 34060 Montpellier, France

(Reçu le 17 décembre 1975, accepté le 19 mars 1976)

\begin{abstract}
Résumé. - Nous présentons ici des résultats expérimentaux et une analyse théorique portant sur la dynamique de la première ligne de Grandjean-Cano sous champ magnétique faible. Le processus dissipatif est essentiellement dû à la réorientation du directeur lors du mouvement de la ligne ce qui nous a conduit à étudier de façon très détaillée la configuration autour de la ligne à partir du modèle planaire de de Gennes. Nous analysons ensuite la dynamique de la ligne. La comparaison entre les résultats théoriques et expérimentaux permet d'atteindre, entre autres paramètres physiques, le rayon du cour de la disclinaison dont l'ordre de grandeur est du domaine macroscopique.
\end{abstract}

\begin{abstract}
Experimental results and theoretical analysis of the dynamics of the first GrandjeanCano line under a weak applied magnetic field are presented here. The dissipative process is essentially due to the director reorientation following the line motion. We therefore give a detailed study of the director configuration around the line, starting from de Gennes planar model. The dynamics of the line is then analysed and compares well with the experimental results. A macroscopic disclination core radius $(\simeq 0,5 \mu)$ is deduced from this comparison.
\end{abstract}

1. Introduction. - It is well known that a magnetic field can distort the usual structure of a cholesteric liquid crystal with positive magnetic anisotropy $\left(\chi_{\mathrm{a}}>0\right)[1,2]$. A static theory of the resulting variation of the pitch $P$ has been developed by de Gennes [3] and Meyer [4]. In experimental verifications performed by the Orsay [5] and Harvard [6] groups, the pitch was derived from the equilibrium position of the Grandjean-Cano lines in a Cano wedge.

However, a dynamical study of the lines motion has not yet been undertaken. In a recent letter [7] we briefly presented the main results concerning the dynamics of the first single line under weak magnetic fields. The purpose of the present paper is to develop in a more detailed and complete version, the experimental and theoretical results on this problem. It is worth pointing out that a similar study has been independently performed by Geurst et al. [8] in twisted nematics.

The effect of a magnetic field normal to the helical axis is double : i) the helix is distorted, ii) the pitch is changed and, as a consequence, in a Cano wedge, the Grandjean-Cano lines move. The first process has been already analysed [9] and we have also recently published a theoretical analysis on this problem [10].

(*) Laboratoire associé au C.N.R.S. (LA 233).
We have found relaxation times for the reorientation of the director of the order of $10^{-1} \mathrm{~s}$. We intend here to analyse the second process. Our experimental results give relaxation times for the line motion of the order of $10^{2} \mathrm{~s}$. We shall therefore make the a priori assumption that these two processes can be treated independently, i.e. that the fast reorientation process is completely achieved when the motion of the lines begins. This means that, in the second process, the energy dissipation is due only to the friction of the line itself. This friction is due to the reorientation of the director in the immediate neighbourhood of the line. To evaluate it, we need a detailed knowledge of the director configuration around the line. This analysis is based upon the de Gennes planar model.

In section 2 the experimental apparatus is described. In section 3 the static theory is presented; this theory leads to the equilibrium conformation of the molecules around the disclination, and the equilibrium position of the line under weak magnetic fields. In section 4, the balance between the change in freeenergy and the dissipation leads to the equation of motion for the line. In section 5 , the experimental results are given and compared with the theoretical predictions.

2. Experiments. -2.1 THE CHOLESTERIC SAMPle. - For this experimental study we need a cholesteric 
material having a positive magnetic anisotropy $\chi_{\mathrm{a}}$. Moreover the experiments are much easier when the sample is cholesteric at room temperature and shows regular Grandjean-Cano lines. Finally in order to have reasonable critical field values $H_{\mathrm{c}}$ for the cholesteric-nematic transition one has to work with large pitch cholesterics.

Among the cholesterol esters, the cholesterol cinnamate of formula

$$
\mathrm{C}_{6} \mathrm{H}_{5}-\mathrm{CH}=\mathrm{CH}-\mathrm{CO}-\mathrm{O}-\mathrm{C}_{27} \mathrm{H}_{45}
$$

gives rise to a stable cholesteric mesophase between $156^{\circ} \mathrm{C}$ and $197^{\circ} \mathrm{C}$. This material has the advantage to show a set of beautiful and regular GrandjeanCano lines [11]. But cholesterol esters usually have a negative anisotropy ; we therefore have used the classical procedure to obtain cholesterics with positive $\chi_{\mathrm{a}}$ : the chiral molecules were dissolved in a conventional nematic.

The equimolar mixture of 4-methoxy-4'-pentyl tolane (MPT) with formula

$$
\mathrm{C}_{5} \mathrm{H}_{11}-\mathrm{C}_{6} \mathrm{H}_{4}-\mathrm{C} \equiv \mathrm{C}-\mathrm{C}_{6} \mathrm{H}_{4}-\mathrm{OCH}_{3}
$$

and 4-propoxy-4'-heptyl tolane (PHT) of formula

$$
\mathrm{C}_{7} \mathrm{H}_{15}-\mathrm{C}_{6} \mathrm{H}_{4}-\mathrm{C} \equiv \mathrm{C}-\mathrm{C}_{6} \mathrm{H}_{4}-\mathrm{OC}_{3} \mathrm{H}_{7}
$$

gives a very stable nematic phase at room temperature [12]. Thus we have dissolved the cholesterol cinnamate in this nematic matrix.

The pitch $P_{0}$ and the cholesteric concentration $c$ are related by the law $[13,14]$ :

$$
P_{0} c=\text { const. }
$$

In order to get large pitches, we have to choose very small cholesteric concentrations :

$$
0,57 \% \leqslant c \leqslant 0,64 \% \text {. }
$$

2.2 The Cano Wedge. - The liquid crystal is introduced in a Cano wedge comprised of a cylindrical lens and a flat plate [13]. The surfaces of the glasses are monitored under the microscope by means of an interferential method which gives also the radius of the lens :

$$
R=80 \pm 1 \mathrm{~mm}
$$

The glasses are cleaned in a sulfochromic mixture, and then precoated with thin films of $\mathrm{SiO}$ evaporated under oblique incidence, in order to obtain a strong anchoring with the easy axis normal to the contact generator [15].

The Cano wedge is fixed in a cell (made of brass) in order to obtain a better contact between the lens and the flat plate.

The pitch is derived from the microscope measurements of the positions of the Grandjean-Cano lines on both sides of the contact generator. For the cholesteric samples used, the unperturbed pitches are :

$$
33,1 \mu \geqslant P_{0} \geqslant 29,5 \mu
$$

with an absolute incertainty of $0,5 \mu$.

2.3 EXPERIMENTAL APPARATUS. - The magnetic field is obtained from a water cooled conventional magnet (with variable air-gap), fed by a stabilized supply. For a 30 A maximal electric current and an $5 \mathrm{~cm}$ air-gap, the magnet gives a $10^{4} \mathrm{G}$ field. The magnetic field is deduced from the electric current measurements. The field calibration was performed by means of a Hall effect gaussmeter.

We use a modified Leitz microscope. Modifications were needed for use in a strong magnetic field. All magnetic pieces were removed and replaced by non-magnetic ones. Adaptation pieces for use in the magnet were also provided. A micro-extension piece supplied with an antivibration device for the adaptation of a Leica camera and a posemeter, are fixed on the top of the microscope.

2.4 Measurements. - The Cano wedge is placed with the easy axis parallel to the magnetic field. The sample is lit by a monochromatic collimated beam and the positions of the Grandjean-Cano lines are observed with different objectives, and by means of a micrometer eye-piece. For each of the three objectives, one division of the micrometer corresponds respectively to $34,0 \pm 0,2 \mu, 10,70 \pm 0,07 \mu, 6,25 \pm 0,02 \mu$.

The unperturbed pitch was controlled before every experiment, and, for each value of the applied magnetic field, a photographic survey is performed at fixed times (generally every fifteen seconds on the beginning, then every half a minute and finally every minute).

Experimental studies have been achieved on four single lines and three double lines under different values of the applied magnetic field $H \lessgtr H_{\mathrm{c}}$ and in cases of increasing and decreasing fields. The corresponding curves giving the displacement versus time are very regular.

Figure 1 shows a set of lines for three values of the magnetic field, and figure 2, corresponds to a field superior to the critical one.

We will give in this paper (section 5) only the results concerning the first single line, and compare them to the theoretical analysis that will be developed in the next two sections. Theoretical and experimental results concerning the other lines will be published later.

3. Static theory. - 3.1 Planar Structure of The FIRST SINGLE GRANDJEAN-CANO LINE. - 3.1.1 The Grandjean-Cano lines. - A cholesteric liquid crystal, placed in a variable thickness cell, shows regular striations which can be easily observed under the microscope.

In the pioneering observations by Grandjean [16] these defects, first identified as walls, were obtained 


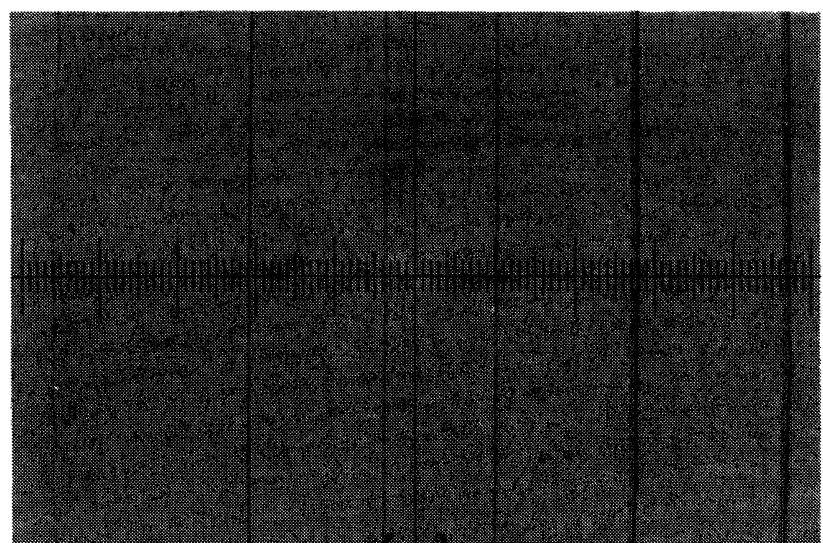

(a)

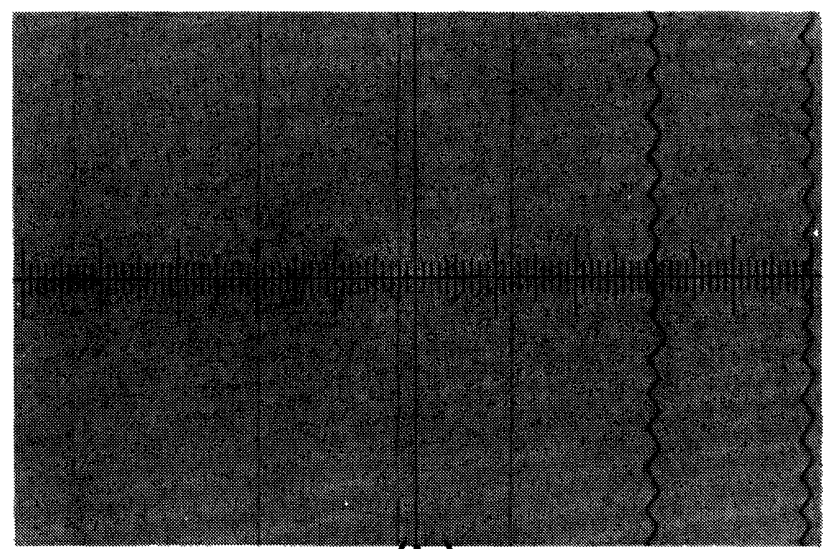

(b)

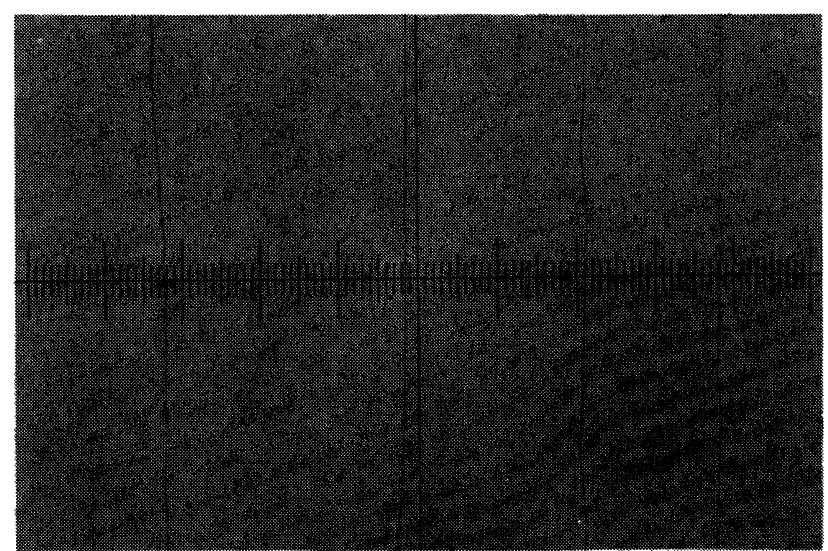

(c)

FIG. 1. - Set of Grandjean-Cano lines for three values of the applied magnetic field $H$. The cholesteric sample is a mixture MPT-PHT, cholesterol cinnamate, $c=0.57 \% P_{0}=33.1 \mu \mathrm{m}$. The contact generator is on the left of the photographs. One division of the micrometer corresponds to $34 \mu \mathrm{m}$. a) Four single lines and two double lines at $H=0 . b$ ) Equilibrium position of the set of lines at $H=5120 \mathrm{G}$. The double lines are deformed into typical zig-zags. c) Position of the lines at $H=7600 \mathrm{G}$ four minutes after applying the field (the initial field is $H_{0}=7200 \mathrm{G}$ ). The two double lines have already disappeared from the photograph.

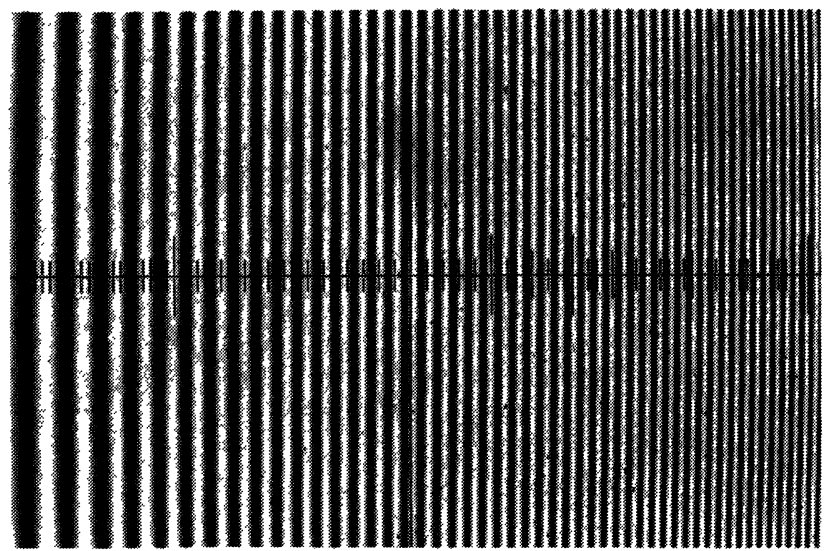

FIG. 2. $-H>H_{\mathrm{c}}$; typical double refraction fringes of the nematic liquid monocrystal between crossed polarizers.

with a cleavage gap inside a sheet of mica. It had before pointed out by Friedel [17] that these walls were in fact threads corresponding to twisting discontinuities, in the helical structure of the cholesteric. In more recent studies Cano [13] inserted the cholesteric sample between rubbed cylindrical lens and flat plate (Cano wedge) and obtained regular threads ordered in parallel lines; by measurements of optical rotatory power, that author [18] has successfully verified that the discontinuities correspond to torsion jumps of one half pitch in helicoidal texture. These results were confirmed by Kassubeck and Meier [19]. In fact, one must distinguish, when one uses thicker wedges, two kinds of lines : thin threads (single lines) which correspond to Cano's work and thick threads (double lines) as shown by the Montpellier and Orsay groups $[20,21]$; the latter threads allow torsion jumps corresponding to two half-pitches jumps.

Two models have been proposed to interpret the singular lines which appear in a Cano wedge : a planar configuration previously described by de Gennes [22], and an application of Volterra process performed by Friedel and Kleman [23].

A very detailed paper on these Grandjean-Cano lines was recently published by Bouligand [24]. He showed that de Gennes model applies to the first single line (and for the other single ones when they are moving), and the Kleman-Friedel model to double lines.

Using de Gennes planar model we will now give a detailed description of the configuration of the molecules around the first thin Grandjean-Cano line.

\subsubsection{Detailed configuration of the molecules around} the first Grandjean-Cano line. - The first GrandjeanCano line appears near the contact generator of a Cano wedge.

In a Cano wedge, the two limiting surfaces are not parallel. However, we will later show that the perturbation of the cholesteric configuration due to the disclination has a range of the order of $d$ along the $x$-axis, where $d$ is the local thickness of the edge. 
As the thickness variation is very smooth, one can neglect the $x$-variation of $d$ over this range and make the assumption that the boundaries are parallel plates. This indeed is true as long as we look at the static configuration of the line. When the line moves, freeenergy variation and dissipation are functions of $d$, which in turn depends on $x$.

It is obvious, from symmetry considerations, that the line should be parallel to the contact generator. A less obvious assertion is that the line is located at equal distances from the two plates. From symmetry arguments one can only say that, if the minimum energy configuration is not the symmetric one, there are two symmetric configurations which minimize the free-energy. However a detailed calculation of the energy versus the line position, which will not be given here, shows that the minimum energy configuration corresponds effectively to the symmetric one.

We have thus the following geometry : the line lies along the $y$-axis and the sample is limited by two planes $z= \pm d / 2$. This is exactly the geometry of de Gennes model : take the helical axis along the $z$-axis and call $\varphi(x, z)$ the twist angle; the director has components :

$n_{x}=\cos \varphi(x, z) ; \quad n_{y}=\sin \varphi(x, z) ; \quad n_{z}=0$.

Like de Gennes, we will assume our cholesteric to be isotropic, i.e. $K_{1}=K_{2}=K_{3}$, where $K_{i}$ are the elastic Frank constants. In fact taking $K_{2}=K_{1}$ (or $K_{3}$ ) can be done with a convenient scaling of the $x$-axis. The only physical assumption is $K_{1}=K_{3}$, which is not exactly the case for real cholesterics where $\left(K_{1}-K_{3}\right) /\left(K_{1}+K_{3}\right)$ is of the order of $\frac{1}{3}$.

Then, minimizing Frank's free-energy, one finds, for the equilibrium condition

$$
\Delta \varphi=0 .
$$

The line separates two regions characterized at large distances by a nematic configuration on the left side and an helicoidal configuration with pitch $2 d$ on the right side (Fig. 3).

Then we have the following boundary conditions, assuming strong anchoring at walls :

$$
\left.\begin{array}{ll}
\varphi=0 \quad \text { at } z=0, z= \pm d / 2 & \text { when } x<0 \\
\varphi=0 & \text { at } z=-d / 2 \\
\varphi=\pi / 2 \text { at } z=0 \\
\varphi=\pi & \text { at } z=d / 2
\end{array}\right\} \quad \text { when } x>0 .
$$

Taking into account these boundary relations (3.3), the partial differential equation can be solved using the technique of conformal mapping. This calculation is performed in appendix A, and the result is :

$$
\begin{aligned}
\varphi(x, z)=\frac{\pi}{2} E(x) & +\frac{\pi z}{2 d}+ \\
+ & \frac{1}{2} \operatorname{Arctan}\left(\operatorname{coth} \frac{\pi x}{d} \tan \frac{\pi z}{d}\right)
\end{aligned}
$$

where $E(x)$ is the Heaviside step function $(E(x)=0$ for $x<0$ and $E(x)=1$ for $x>0$ ).

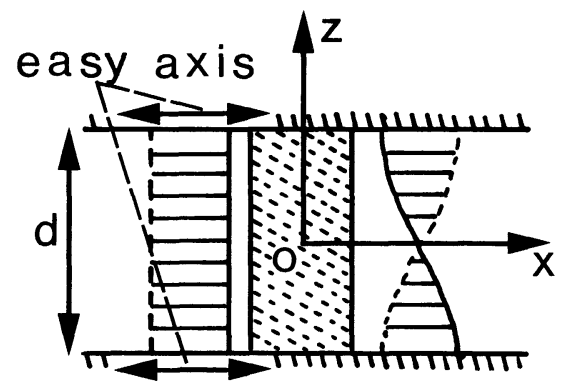

FIG. 3. - The region around $O z$-axis is the connection zone between nematic and cholesteric regions. The thickness $d$ is assumed to be constant. The distortion range is $d / \pi$.

The main characteristics of the configuration described by eq. (3.4) are the following ones :

1) Far from the line $(x \rightarrow \pm \infty)$

$$
\begin{array}{rlrl}
x \gg \frac{d}{\pi}, \varphi & =\frac{\pi}{2}+\frac{\pi z}{d} & & \text { (cholesteric) } \\
x \gg-\frac{d}{\pi}, \varphi & =0 & \text { (nematic) } .
\end{array}
$$

2) On the plates

$$
\begin{aligned}
& z=-\frac{d}{2}, \quad \varphi=0 \\
& z=\frac{d}{2}, \quad \varphi=0 \text { for } x<0 ; \varphi=\pi \text { for } x>0
\end{aligned}
$$

Note that the $\pi$ discontinuity has no physical meaning.

3) In the central horizontal plane $(z=0)$

$$
\begin{aligned}
& \varphi=0 \text { for } x<0 \\
& \varphi=\frac{\pi}{2} \text { for } \quad x>0 .
\end{aligned}
$$

This discontinuity is classical in de Gennes model. 4) In the central vertical plane $(x=0)$

$$
\begin{aligned}
& \text { for } z>0\left\{\begin{array}{l}
x \rightarrow 0_{+}, \varphi \rightarrow \frac{3 \pi}{4}+\frac{\pi z}{2 d} \\
x \rightarrow 0_{-}, \varphi \rightarrow \frac{3 \pi}{4}+\frac{\pi z}{2 d}-\pi
\end{array}\right. \\
& \text { for } z<0, \quad \varphi=\frac{\pi}{4}+\frac{\pi z}{2 d} .
\end{aligned}
$$

Here again the $\pi$ discontinuity for $z>0$ has no physical meaning. On the other hand the line introduces a $\pi / 2$ discontinuity which was not pointed out before.

The general configuration is given in figures 4 and 5 . Starting from the right hand side, one finds at long 
range, a regular half helix (region $B$ ) whose spatial period is $L=d$ (3.5). Drawing near to the yoz plane, the cholesteric spiral will distort; nearest this plane one finds a regular helicoidal structure of double spatial period (region $\mathrm{B}^{\prime}$ ), except close the xoy plane where the twist angle abruptly increases form $\pi / 4$ to $3 \pi / 4$, (3.11) (3.13), with a value $\pi / 2$ at $z=0$ (3.10). This steep increase tends to a $\pi / 2$ jump at $x=0_{+}$.

Starting now from the left hand side, the liquid crystal is nematic at long range (3.6) (region A). Drawing near to the $y o z$ plane the nematic structure will distort, and close to this plane one has again a regular twist distortion (region $\mathrm{A}^{\prime}$ ) corresponding to the spatial period $2 d$, except near the xoy plane where a sudden twist reversal appears, the twist angle annealing in the xoy plane (3.9); here we get a $-\pi / 2$ jump (from $\pi / 4$ to $-\pi / 4$ ) at $x=0_{-}$(3.12) (3.13).

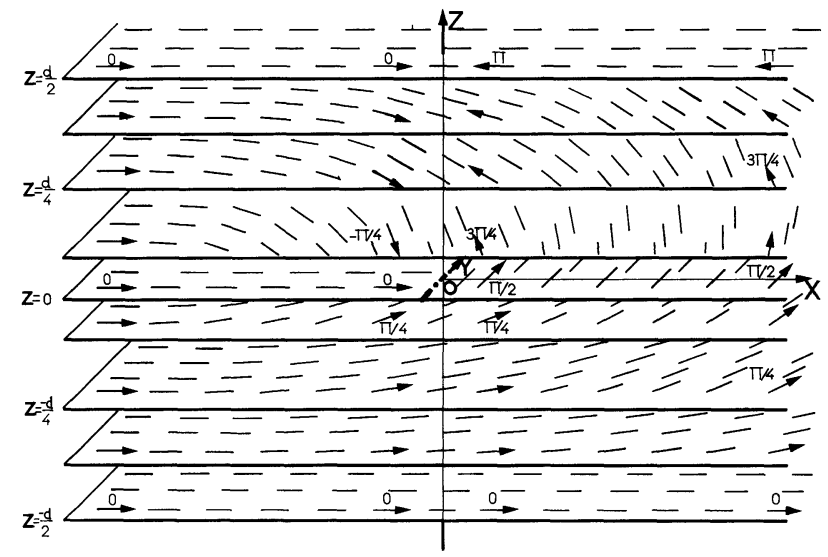

FIG. 4. - Planar configuration of the molecules around the first thin line. The core of this line lies along the $O y$ direction. The upper and lower planes nearest the plane $z=0$ are infinitely close to the latter. The numbers represent the different values of the tilt angle $\varphi(x, z)$ between the director (arrows) and the $x$-axis.

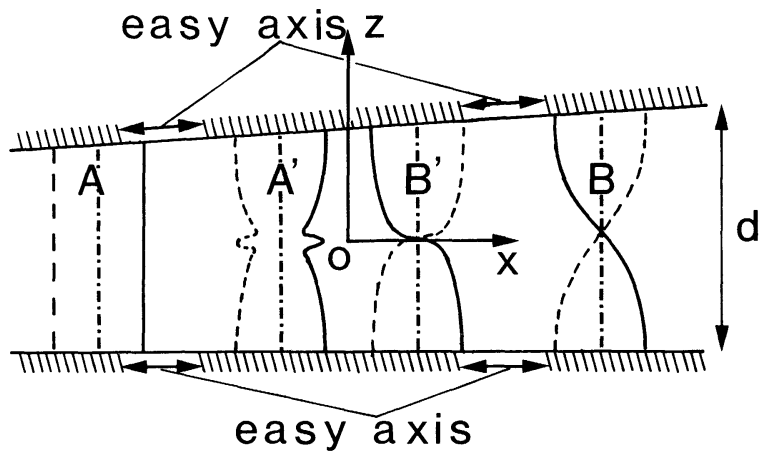

FIG. 5. - Details of the perturbed field around the first single line : the nematic configuration (region A) and the cholesteric helicoidal one whose spatial period is $L=d$ (region $\mathrm{B}$ ) are separated by the connection zone. This zone is characterized on both sides of the line by a helical structure of spatial period $L=2 d$, which exhibits a typical jump of torsion respectively opposed and occurring close to the xoy plane (regions $\mathrm{A}^{\prime}$ and $\mathrm{B}^{\prime}$ ). The full lines represent the head of the arrows drawn on figure 5, and the dotted ones the end of these arrows.
Twist reversal. $-\partial \varphi / \partial z$ is easily derived from eq. (3.4) :

$$
\frac{\partial \varphi}{\partial z}=\frac{\pi}{2 d}\left[1+\frac{\sinh \frac{2 \pi x}{d}}{\cosh \frac{2 \pi x}{d}-\cos \frac{2 \pi z}{d}}\right]
$$

twist reversal then occurs for

$$
\cos \frac{2 \pi z}{d}=\exp \left(\frac{2 \pi x}{d}\right) .
$$

Eq. (3.14) can be satisfied only for $x<0$. The twist reversal surface is defined therefore by

$$
z= \pm z_{\mathrm{r}}(x)= \pm \frac{d}{2 \pi} \operatorname{Arccos}\left(\exp \frac{2 \pi x}{d}\right)
$$

The corresponding twist angle is given by

$$
\varphi=\frac{\pi z_{\mathrm{r}}}{2 d}-\frac{1}{2} \operatorname{Arctan}\left(\cot \frac{\pi z_{\mathrm{r}}}{d}\right)
$$

i.e., for $z<0$

$$
\varphi_{\max }=-\frac{\pi z_{\mathrm{r}}}{d}+\frac{\pi}{4}
$$

and for $z>0$

$$
\varphi_{\min }=\frac{\pi z_{\mathrm{r}}}{d}-\frac{\pi}{4}
$$

Hence the twist reversal is steep for small values of $z_{\mathrm{r}}$, i.e. for $|x| \ll \pi / d$.

It extends over a range $\pi / d$ along the $x$ direction.

3.2 EQUILIBRIUM POSITION OF THE FIRST SINGLE GRANDJEAN-CANO LINE UNDER APPLIED MAGNETIC FIELD. - In a Cano wedge, the cholesteric liquid crystal is inserted between a cylindrical lens and a flat plate (Fig. 6). The frame of reference is the same as above and the cholesteric material has a positive magnetic anisotropy $\left(\chi_{\mathrm{a}}>0\right)$.

When a static magnetic field $H$ is applied along the $x$ direction (i.e. normal to the helical axis $O z$ ), the pitch increases and, as a consequence, the Grandjean-Cano line moves to a new equilibrium position.

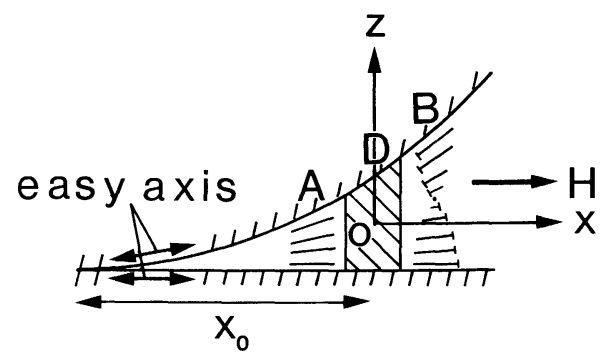

FIG. 6. - Cholesteric sample in cylindrical geometry (Cano wedge). The equilibrium position of the first Grandjean-Cano line, under the initial magnetic field $H_{0}$, is assumed to be along the $y$-axis. The distorted region (D) separates the nematic (A) and cholesteric (B) configurations. 
We will now derive this equilibrium position from the minimization of the free-energy in presence of the applied magnetic field. The sample can be divided into three regions :

- left of the line, the sample is nematic (region A),

- in a range $d$ around the line, the sample has the distorted configuration previously described (region D),

- on the right of the line, the sample has a distorted cholesteric configuration (region B).

The latter distortion has been described in a previous publication [10]. In this earlier paper, only twist deformations were taken into account. However, as the variation of $d$ is very smooth, one can easily show that the bend and splay terms in the free-energy expression due to the variation of $d$ can be neglected in regard of the twist and magnetic terms.

When the field increases, region $\mathrm{D}$ moves to the right. Its free-energy can be assumed to be constant. As the distortion range is proportional to the width $d$ of the sample, one can assume that the core radius is also proportional to $d$. As the core radius is taken into account only through its logarithm (appendix B), this is not a very severe restriction.

Then the change in free-energy is due to the extension of region $\mathrm{A}$, and to the subsequent decrease of region $B$.

The energy per unit-surface area of region $\mathrm{A}$ is :

$$
F_{N}=\frac{K_{2}}{2}\left(q_{0}^{2} d-\frac{d}{\xi^{2}}\right)
$$

where $\xi$ is the magnetic coherence length :

$$
\xi=\frac{1}{H}\left(\frac{K_{2}}{\chi_{\mathrm{a}}}\right)^{1 / 2} .
$$

The energy per unit surface of region $B$ is [10] :

$F_{\mathrm{C}}=\frac{K_{2}}{2}\left[q_{0}^{2} d-2 \pi q_{0}-\frac{d}{k^{2} \xi^{2}}+\frac{4}{k \xi} E(k)\right]$

here $E(k)$ is the complete elliptic integral of the second kind [25] and its modulus $k$ is defined by the implicit equation :

$$
k K(k)=\frac{d}{2 \xi}
$$

where $K(k)$ is the complete elliptic integral of the first kind.

In eqs. $(3.15,16$ and 17), $d$ is a function of $x$. Take the $y$-axis at the initial position of the line (under the initial applied field $H_{0}$ ). Then $d$ is given by :

$$
d(x)=\frac{\left(x+x_{0}\right)^{2}}{2 R}
$$

where $R$ is the lens radius, $x_{0}$ the distance between the contact generator and the $y$-axis.
Let $x_{\mathrm{L}}$ be the position of the line, then the total free-energy is :

$$
F=\int_{-x_{0}}^{x_{\mathrm{L}}} F_{N}[d(x)] \mathrm{d} x+F_{\mathrm{D}}+\int_{x_{\mathrm{L}}}^{\infty} F_{\mathrm{C}}[d(x)] \mathrm{d} x
$$

where $F_{\mathrm{D}}$ is the excess free-energy of the strong distortion due to the disclination line $\left(F_{\mathrm{D}}\right.$ is calculated in appendix B). Then the equilibrium condition is :

$$
\frac{\mathrm{d} F}{\mathrm{~d} x_{\mathrm{L}}}=F_{N}\left[d\left(x_{\mathrm{L}}\right)\right]-F_{\mathrm{C}}\left[d\left(x_{\mathrm{L}}\right)\right]=0
$$

which gives, using eqs. (3.15 and 16)

$$
\frac{K_{2}}{\xi}\left[\pi q_{0} \xi-\frac{k^{2}-1}{k^{2} \xi} d-\frac{2}{k} E(k)\right]=0
$$

or using eq. (3.17) :

$$
\pi q_{0} \xi=\frac{2 E(k)-k^{2} K(k)}{k}
$$

where $k^{\prime 2}=1-k^{2}$.

Note that eq. (3.21) provides the de Gennes-Meyer formula for the critical field of the cholesteric-nematic transition :

From eq. (3.17), $d$ diverges for $k=1$; then using :

$$
E(1)=1, \quad \lim _{k \rightarrow 1} K(k)=\ln \frac{4}{k^{\prime}},
$$

one gets :

$$
\pi q_{0} \xi_{\mathrm{c}}=2
$$

which gives the classical value for the reduced critical field $h_{\mathrm{c}}$ :

$$
h_{\mathrm{c}}=\frac{1}{q_{0} \xi_{\mathrm{c}}}=\frac{\pi}{2} .
$$

Moreover, for $H=0, k=0$, one finds the usual relation :

$$
d=\frac{\pi}{2 q_{0}}=\frac{P_{0}}{4} .
$$

In the case of weak magnetic fields, $k \rightarrow 0 . E(k)$ and $K(k)$ can then be expanded in series in $k$ :

$$
E(k) \simeq \frac{\pi}{2}\left(1-\frac{k^{2}}{4}\right), \quad K(k) \simeq \frac{\pi}{2}\left(1+\frac{k^{2}}{4}\right),
$$

then eqs. (3.20) and (3.21) reduce to :

$$
\begin{aligned}
k & \simeq \frac{d}{\pi \xi}\left(1-\frac{d^{2}}{4 \pi^{2} \xi^{2}}\right) \\
q_{0} & \simeq \frac{\pi}{2 d}\left(1+\frac{d^{2}}{2 \pi^{2} \xi^{2}}\right) .
\end{aligned}
$$


The local thickness corresponding to the equilibrium position of the line is then given by :

$$
d \simeq \frac{\pi}{2 q_{0}}\left(1+\frac{1}{8 q_{0}^{2} \xi^{2}}\right) .
$$

It is worth noticing that eq. (3.23) gives for $H=0$, $d=\pi / 2 q_{0}$ i.e. $d=P_{0} / 4$, which effectively gives the position of the first Grandjean-Cano line in the absence of magnetic field.

4. Dynamics. - 4.1 Differential eQUATION FOR THE LINE MOTION. - The dynamics of the line will be derived from the energy conservation law. We have already shown that the change in free-energy is essentially bound to the line motion (section 3 eq. (3.20))

$$
\frac{\mathrm{d} F}{\mathrm{~d} t}=\frac{\mathrm{d} F}{\mathrm{~d} x_{\mathrm{L}}} \frac{\mathrm{d} x_{\mathrm{L}}}{\mathrm{d} t}=\left[F_{\mathrm{n}}\left(x_{\mathrm{L}}\right)-F_{\mathrm{c}}\left(x_{\mathrm{L}}\right)\right] \frac{\mathrm{d} x_{\mathrm{L}}}{\mathrm{d} t} .
$$

This free-energy variation will be balanced by the entropy production bound to the reorientation of the director. This reorientation takes place in region (D), (Fig. 6), i.e. in a range $d$ around the disclination line. It involves a twist rotation, which is well known to be uncoupled to the hydrodynamic motion (this can be easily deduced from Leslie's eq. [26]).

Then the expression for the entropy source is :

$$
T \dot{S}=\int_{-\infty}^{+\infty} \mathrm{d} x \int_{-d / 2}^{d / 2} \gamma_{1}\left(\frac{\mathrm{d} \varphi}{\mathrm{d} t}\right)^{2} \mathrm{~d} z
$$

where $\gamma_{1}$ is a Leslie friction coefficient.

We must here point out that, in this expression, $d$ should be a function of $x$. However, as the effective range of the disclination is $d / \pi$, the integrand has a significant value only for $x_{\mathrm{L}}-d<x<x_{\mathrm{L}}+d$, and we can neglect the variation of $d$ in this interval. As a consequence, we can consider $d$ as constant in the integration of the r.h.s. of eq. (4.2).

Around the disclination, $\varphi$ is a function of $\left(x-x_{\mathrm{L}}, z\right)$, $\mathrm{d} \varphi / \mathrm{d} t$ is the total time derivative of $\varphi(\mathrm{M})$ where the point $\mathbf{M}(x, z)$ is fixed in the laboratory frame of reference. Then,

$$
\frac{\mathrm{d} \varphi}{\mathrm{d} t}=-\frac{\partial \varphi}{\partial\left(x-x_{\mathrm{L}}\right)} \frac{\mathrm{d} x_{\mathrm{L}}}{\mathrm{d} t}
$$

and eq. (4.2) can be rewritten :

$$
T \dot{S}=\gamma_{1}\left(\frac{\mathrm{d} x_{\mathrm{L}}}{\mathrm{d} t}\right)^{2} \int_{-\infty}^{+\infty} \mathrm{d} x \int_{-d / 2}^{d / 2}\left(\frac{\partial \varphi}{\partial x}\right)^{2} \mathrm{~d} z
$$

The expression for $\partial \varphi / \partial x$ is derived in appendix A. One finds :

$$
\frac{\partial \varphi}{\partial x}=-\left(\frac{K_{2}}{K}\right)^{1 / 2} \frac{\pi}{2 d} \frac{\sin \frac{2 \pi z}{d}}{\cosh \left(\frac{K_{2}}{K}\right)^{1 / 2} \frac{2 \pi x}{d}-\cos \frac{2 \pi z}{d}}
$$

where $K=K_{1}=K_{3} \neq K_{2}$.
As mentioned above, $\partial \varphi / \partial x$ has a significant value only near the disclination, which allows well for our previous assumption $d=$ const. in the integration of eq. (4.3).

Now define the dissipation integral :

$$
V(d)=\int_{-\infty}^{+\infty} \mathrm{d} x \int_{-d / 2}^{d / 2}\left(\frac{\partial \varphi}{\partial x}\right)^{2} \mathrm{~d} z .
$$

Eq. (4.3) now reads :

$$
T \dot{S}=\gamma_{1}\left(\frac{\mathrm{d} x_{\mathrm{L}}}{\mathrm{d} t}\right)^{2} V(d) .
$$

The next step is to write the energy balance equation :

$$
T \dot{S}=-\frac{\mathrm{d} F}{\mathrm{~d} t}
$$

In this expression, we must now take $d$ as a function of $x_{\mathrm{L}}$.

Eq. (4.7) is the differential equation for the motion of the line.

A more explicit expression for the r.h.s. of eq. (4.7) can be written in the weak field approximation. We must here point out that the detailed configuration around the line has been obtained in the zero-field case. In fact, this approximation holds as long as the distortion range $d / \pi$ is smaller than the magnetic coherence length $\xi$, i.e. for magnetic fields smaller than the critical field $H_{\mathrm{c}}\left({ }^{1}\right)$. Then, in the weak field approximation, one has [10] :

$$
F_{\mathrm{c}} \simeq \frac{K_{2}}{2} d\left[\left(\frac{\pi}{d}-q_{0}\right)^{2}-\frac{1}{2 \xi^{2}}\right]
$$

and eq. (4.1) becomes :

$$
\frac{\mathrm{d} F}{\mathrm{~d} t}=\frac{K_{2}}{2}\left[-\frac{\pi^{2}}{d}+2 \pi q_{0}-\frac{d}{2 \xi^{2}}\right] \frac{\mathrm{d} x_{\mathrm{L}}}{\mathrm{d} t} .
$$

Using eqs. (4.6) and (4.8), eq. (4.7) becomes

$$
\frac{\gamma_{1}}{\chi_{\mathrm{a}} H^{2}} \frac{\mathrm{d} X}{\mathrm{~d} t} V(d)=\xi^{2} \frac{\pi^{2} R}{X^{2}}-\xi^{2} \pi q_{0}+\frac{X^{2}}{8 R}
$$

where one has replaced $d$ by the expression (3.18), using the notation

$$
X=x_{\mathrm{L}}+x_{0} .
$$

Define now the following reduced variables :

$X^{\prime}=\frac{X}{\xi}, \quad t^{\prime}=\frac{\chi_{\mathrm{a}} H^{2} t}{\gamma_{1}}, \quad r=\frac{R}{\xi}, \frac{1}{h}=q_{0} \xi$.

( ${ }^{1}$ ) One must have $\left(\frac{\partial \varphi}{\partial z}\right)_{\text {magn. }} \dot{\ddot{*}}\left(\sim \frac{d}{4 \pi \xi^{2}}\right) \ll\left(\frac{\partial \varphi}{\partial z}\right)_{\text {dist. }}\left(\sim \frac{\pi}{2 d}\right)$; this implies that $d^{2} / \xi^{2} \ll 2 \pi^{2}$ or $\left(H / H_{\mathrm{c}}\right)^{2} \ll 32 / \pi^{2}$. 
The differential equation of motion (4.9) of the line takes the form :

$$
\frac{\mathrm{d} X^{\prime}}{\mathrm{d} t^{\prime}} V(d)=\frac{\pi^{2} r}{X^{\prime 2}}-\frac{\pi}{h}+\frac{X^{\prime 2}}{8 r} .
$$

A detailed calculation of the dissipation integral $V(d)$ (eq. (4.5)) is given in appendix B. One gets a logarithmic divergence at the line position and one has to introduce, as a cut-off radius, the core radius of the disclination line, $\varepsilon$. The result is then :

$$
V=\frac{\pi}{4}\left(\frac{K_{2}}{K}\right)^{1 / 2}\left[\ln \frac{d}{4 \pi \varepsilon}\left(\frac{K}{K_{2}}\right)^{1 / 2}+\frac{1}{2}+\frac{2 G}{\pi}\right]
$$

where $G$ is the Catalan's constant : $G \simeq 0.916$. Note that this logarithmic divergence is usual in disclination problems.

4.2 Motion eQuATION OF THE LINE. - We have already assumed in section 3 that the core radius $\varepsilon$ is proportional to the range of the connection zone, and therefore to the local thickness $d$. Thus, in eq. (4.13), the term $\ln \frac{d}{4 \pi \varepsilon}\left(\frac{K}{K_{2}}\right)^{1 / 2}$ can be taken as a constant.

One can now integrate the differential equation (4.12) with $V=$ const., define the following notations :

$$
A=\pi^{2} r, \quad B=-\frac{\pi}{h}, \quad C=\frac{1}{8 r} .
$$

Eq. (4.12) becomes :

$$
\frac{\mathrm{d} t^{\prime}}{V}=\frac{X^{\prime 2} \mathrm{~d} X^{\prime}}{A+B X^{\prime 2}+C X^{\prime 4}} .
$$

Let,

$$
D^{2}=B^{2}-4 A C=\pi^{2}\left(\frac{1}{h^{2}}-\frac{1}{2}\right)>0 .
$$

This condition is satisfied for

$$
h<h_{1} \text { with } h_{1}=\sqrt{2} .
$$

Define now

$$
\begin{gathered}
\alpha=\left(1-\frac{h^{2}}{h_{1}^{2}}\right)^{1 / 2}, \\
G_{ \pm}=\frac{B}{2} \mp \frac{D}{2}=-\frac{\pi}{2 h}(1 \pm \alpha) .
\end{gathered}
$$

Taking into account the boundary condition :

$$
X^{\prime}=\frac{X_{0}}{\xi}=X_{0}^{\prime} \text { for } t=0 .
$$

One can then integrate eq. (4.15) [27] :

$$
\frac{t^{\prime}}{V}=\frac{G_{-}}{D} \int_{X_{0}^{\prime}}^{X^{\prime}} \frac{\mathrm{d} X^{\prime}}{C X^{\prime 2}+G_{-}}-\frac{G_{+}}{D} \int_{X_{0}^{\prime}}^{X^{\prime}} \frac{\mathrm{d} X^{\prime}}{C X^{\prime 2}+G_{+}} .
$$

Note that the products $C G_{-}$and $C G_{+}$are always negative; thus :

$$
\frac{t^{\prime}}{V}=\left.\frac{G_{-}}{D} \frac{1}{2 i \sqrt{C G_{-}}} \ln \frac{G_{-}+i X^{\prime} \sqrt{C G_{-}}}{G_{-}-i X^{\prime} \sqrt{C G_{-}}}\right|_{X_{0}^{\prime}} ^{X^{\prime}}-\left.\frac{G_{+}}{D} \frac{1}{2 i \sqrt{C G_{+}}} \ln \frac{G_{+}+i X^{\prime} \sqrt{C G_{+}}}{G_{+}-i X^{\prime} \sqrt{C G_{+}}}\right|_{X_{0}^{\prime}} ^{X^{\prime}} .
$$

According to eqs. (4.16) and (4.18) one gets :

$$
\begin{aligned}
& \frac{t^{\prime}}{V}=\frac{1}{\alpha \sqrt{\frac{\pi}{r h}}}\left\{\sqrt{1-\alpha}\left[\ln \frac{1+\frac{X^{\prime}}{2} \sqrt{\frac{h}{\pi r(1-\alpha)}}}{1-\frac{X^{\prime}}{2} \sqrt{\frac{h}{\pi r(1-\alpha)}}}-\ln \frac{1+\frac{X_{0}^{\prime}}{2} \sqrt{\frac{h}{\pi r(1-\alpha)}}}{1-\frac{X_{0}^{\prime}}{2} \sqrt{\frac{h}{\pi r(1-\alpha)}}}\right]-\right. \\
& \left.-\sqrt{1+\alpha}\left[\ln \frac{1+\frac{X^{\prime}}{2} \sqrt{\frac{h}{\pi r(1+\alpha)}}}{1-\frac{X^{\prime}}{2} \sqrt{\frac{h}{\pi r(1+\alpha)}}}-\ln \frac{1+\frac{X_{0}^{\prime}}{2} \sqrt{\frac{h}{\pi r(1+\alpha)}}}{1-\frac{X_{0}^{\prime}}{2} \sqrt{\frac{h}{\pi r(1+\alpha)}}}\right]\right\} \text {. }
\end{aligned}
$$

the equilibrium position is obtained for $t=\infty$, i.e. for $X^{\prime}=X_{\mathrm{e}}^{\prime}$ such as :

$$
\frac{X_{\mathrm{e}}^{\prime}}{2} \sqrt{\frac{h}{\pi r(1-\alpha)}}=1
$$

which gives, expressed into the initial variables (eqs. (4.10), (4.11))

$$
d=\frac{\left(x_{\mathrm{e}}+x_{0}\right)^{2}}{2 R}=2 \pi q_{0} \xi^{2}\left[1-\left(1-\frac{1}{2 q_{0}^{2} \xi^{2}}\right)^{1 / 2}\right]
$$


or

$$
d \simeq \frac{\pi}{2 q_{0}}\left(1+\frac{1}{8 q_{0}^{2} \xi^{2}}\right)
$$

which is the expression already found in eq. (3.23).

A detailed discussion of logarithmic terms in eq. (4.19) is given in appendix C. One finds, introducing the functions $\tanh ^{-1}$ and coth ${ }^{-1}$ :

i) In case of increasing fields $\left(H>H_{0}\right)$

$$
\begin{aligned}
\left.\frac{t^{\prime}}{V}=\frac{2}{\alpha \sqrt{\frac{\pi}{r h}}\left\{\sqrt { 1 - \alpha } \left[\tanh ^{-1} \frac{X^{\prime}}{2}\right.\right.} \sqrt{\frac{h}{\pi r(1-\alpha)}}-\tanh ^{-1} \frac{X_{0}^{\prime}}{2} \sqrt{\frac{h}{\pi r(1-\alpha)}}\right]- \\
\left.\quad-\sqrt{1+\alpha}\left[\tanh ^{-1} \frac{X^{\prime}}{2} \sqrt{\frac{h}{\pi r(1+\alpha)}}-\tanh ^{-1} \frac{X_{0}^{\prime}}{2} \sqrt{\frac{h}{\pi r(1+\alpha)}}\right]\right\} .
\end{aligned}
$$

ii) In case of decreasing fields $\left(H<H_{0}\right)$

$$
\begin{aligned}
\frac{t^{\prime}}{V}=\frac{2}{\alpha \sqrt{\frac{\pi}{r h}}\left\{\sqrt { 1 - \alpha } \left[\operatorname{coth}^{-1} \frac{X^{\prime}}{2}\right.\right.} & \left.\sqrt{\frac{h}{\pi r(1-\alpha)}}-\operatorname{coth}^{-1} \frac{X_{0}^{\prime}}{2} \sqrt{\frac{h}{\pi r(1-\alpha)}}\right]- \\
& \left.-\sqrt{1+\alpha}\left[\tanh ^{-1} \frac{X^{\prime}}{2} \sqrt{\frac{h}{\pi r(1+\alpha)}}-\tanh ^{-1} \frac{X_{0}^{\prime}}{2} \sqrt{\frac{h}{\pi r(1+\alpha)}}\right]\right\} .
\end{aligned}
$$

Insert now eqs. $(4.10,11)$ in eqs. $(4.21,22)$ and define the following quantities :

$$
\begin{aligned}
\tau_{ \pm} & =\frac{2 \gamma_{1} V \sqrt{1 \pm \alpha}}{\alpha \chi_{\mathrm{a}} H^{2} \xi \sqrt{\pi q_{0} / R}} \\
l_{ \pm} & =2 \xi \sqrt{q_{0} \pi R(1 \pm \alpha)} .
\end{aligned}
$$

The equations of motion take the forms :

$$
\begin{aligned}
& t=\tau_{-}\left(\tanh ^{-1} \frac{x+x_{0}}{l_{-}}-\tanh ^{-1} \frac{x_{0}}{l_{-}}\right)-\tau_{+}\left(\tanh ^{-1} \frac{x+x_{0}}{l_{+}}-\tanh ^{-1} \frac{x_{0}}{l_{+}}\right), \text {for } H>H_{0} \\
& t=\tau_{-}\left(\operatorname{coth}^{-1} \frac{x+x_{0}}{l_{-}}-\operatorname{coth}^{-1} \frac{x_{0}}{l_{-}}\right)-\tau_{+}\left(\tanh ^{-1} \frac{x+x_{0}}{l_{+}}-\tanh ^{-1} \frac{x_{0}}{l_{+}}\right), \text {for } \quad H<H_{0}
\end{aligned}
$$

and in the last equation $x$ takes evidently negative values.

5. Experimental and theoretical results. 5.1 PitCH Versus MAGNeTIC FIELD. - The experimental variation of the pitch $P$ versus the magnetic field $H$ is given in figure 7. In this experiment the pitch is derived from the equilibrium position of the Grandjean-Cano lines in the Cano wedge. The experimental curve is quite similar to the ones found by several authors $[5,6]$.

From this curve one can deduce the critical field for the cholesteric-nematic transition : the unperturbed pitch here is $P_{0}=29.5 \mu$ and one finds : $H_{\mathrm{c}}=7700 \mathrm{G}$.

The de Gennes-Meyer formula :

$$
H_{\mathrm{c}}=\frac{\pi^{2}}{P_{0}}\left(\frac{K_{2}}{\chi_{\mathrm{a}}}\right)^{1 / 2}
$$

gives then

$$
\frac{K_{2}}{\chi_{\mathrm{a}}}=5.3 \pm 0.3 \text { c.g.s. }
$$

Taking into account the experimental value of $\chi_{\mathrm{a}}\left({ }^{2}\right): \chi_{\mathrm{a}} \simeq 10^{-7}$ u.e.m.c.g.s., one deduces the twist elastic constant :

$$
K_{2} \simeq 5.3 \times 10^{-7} \text { dyne } .
$$

This result is in good agreement with the value found by Sicard [28] on the same material.

5.2 Equilibrium POSITIONS OF THE SINGLE THIN LINE. - The expression for the equilibrium position

$\left({ }^{2}\right)$ Achard, M. F., Sigaud, G., private communication. 


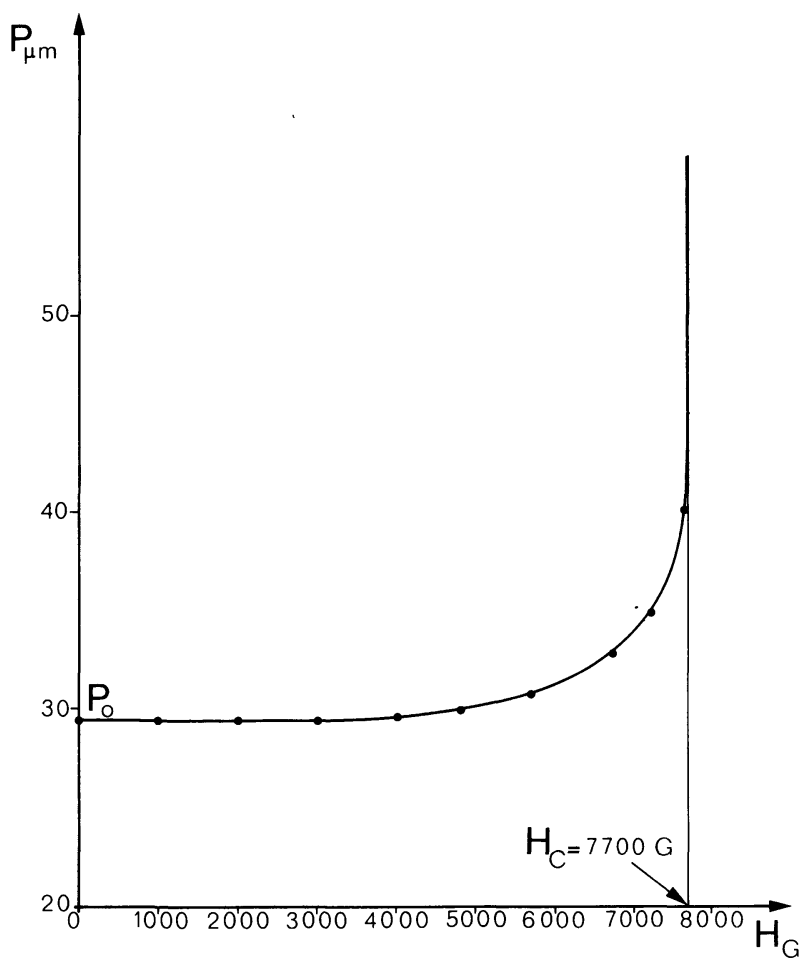

FIG. 7. - Pitch $P$ versus magnetic field $H$. The initial pitch is here $P_{0}=29.5 \mu \mathrm{m}$ and the critical field $H_{\mathrm{c}}=7700 \mathrm{G}$. The black circles are the experimental points.

of the first single line under an applied magnetic field has been given in section 3 (eqs. (3.18), (3.23)). It can rewritten :

$$
X_{\mathrm{e}}=\left[\frac{\pi R}{q_{0}}\left(1+\frac{1}{8 q_{0}^{2} \xi^{2}}\right)\right]^{1 / 2}
$$

where $X_{\mathrm{e}}=x+x_{0}$ is the equilibrium position measured from the contact generator.

This theoretical expression is compared in table I to our experimental results. The measurements have been made both for increasing and decreasing fields. The relative discrepancies

$$
\frac{\Delta X_{\mathrm{e}}}{X_{\mathrm{e}}}=\frac{X_{\mathrm{e}}^{\prime}(\text { theor. })-X_{\mathrm{e}}(\text { exp. })}{X_{\mathrm{e}}(\text { exp. })}
$$

are given in the last column. They do not exceed the experimental accuracy of the measurements.

5.3 MOTION OF THE FIRST THIN LINE UNDER WEAK MAGNETIC FIELDS. - In order to give a physical interpretation of the relaxation times $\tau$ eq. (4.23) occurring in the equations of motion for the line, eqs. (4.25) and (4.26), let us introduce the following parameters :

$$
\begin{gathered}
\xi_{1}=\frac{1}{H_{1}}\left(\frac{K_{2}}{\chi_{\mathrm{a}}}\right)^{1 / 2} \text { where } H_{1}=\frac{2 \sqrt{2}}{\pi} H_{\mathrm{c}} \\
f_{ \pm}=\frac{1}{\alpha(1 \mp \alpha)^{1 / 2}} \quad \text { where } \quad \alpha=\left(1-\frac{H^{2}}{H_{1}^{2}}\right)^{1 / 2}
\end{gathered}
$$

then :

$$
\tau_{ \pm}=\tau_{0} f_{ \pm}\left(H / H_{1}\right)
$$

where

$$
\tau_{0}=\frac{\gamma_{\text {eff }}}{\chi_{\mathrm{a}} H_{1}^{2}}
$$

and

$$
\gamma_{\mathrm{eff}}=\frac{2 V \gamma_{1}}{\xi_{1}\left(\pi q_{0} / R\right)^{1 / 2}} .
$$

$\tau_{0}$ is the field independent relaxation time and $\gamma_{\text {eff }}$ an effective viscosity.

Replacing $\gamma_{\text {eff }}, \xi_{1}, H_{1}$ and $H_{c}$ by their expressions, eq. (5.2) can be rewritten :

$$
\tau_{0}=\frac{\gamma_{1}}{K_{2} q_{0}^{2}} V\left(\frac{2 q_{0} R}{\pi}\right)^{1 / 2} .
$$

$\tau_{0}$ therefore appears to be a purely elastic relaxation time.

The physical meaning of the two relaxation times, $\tau_{+}$and $\tau_{-}$is less evident. We will now show that, for low magnetic fields, their behaviour is quite different.

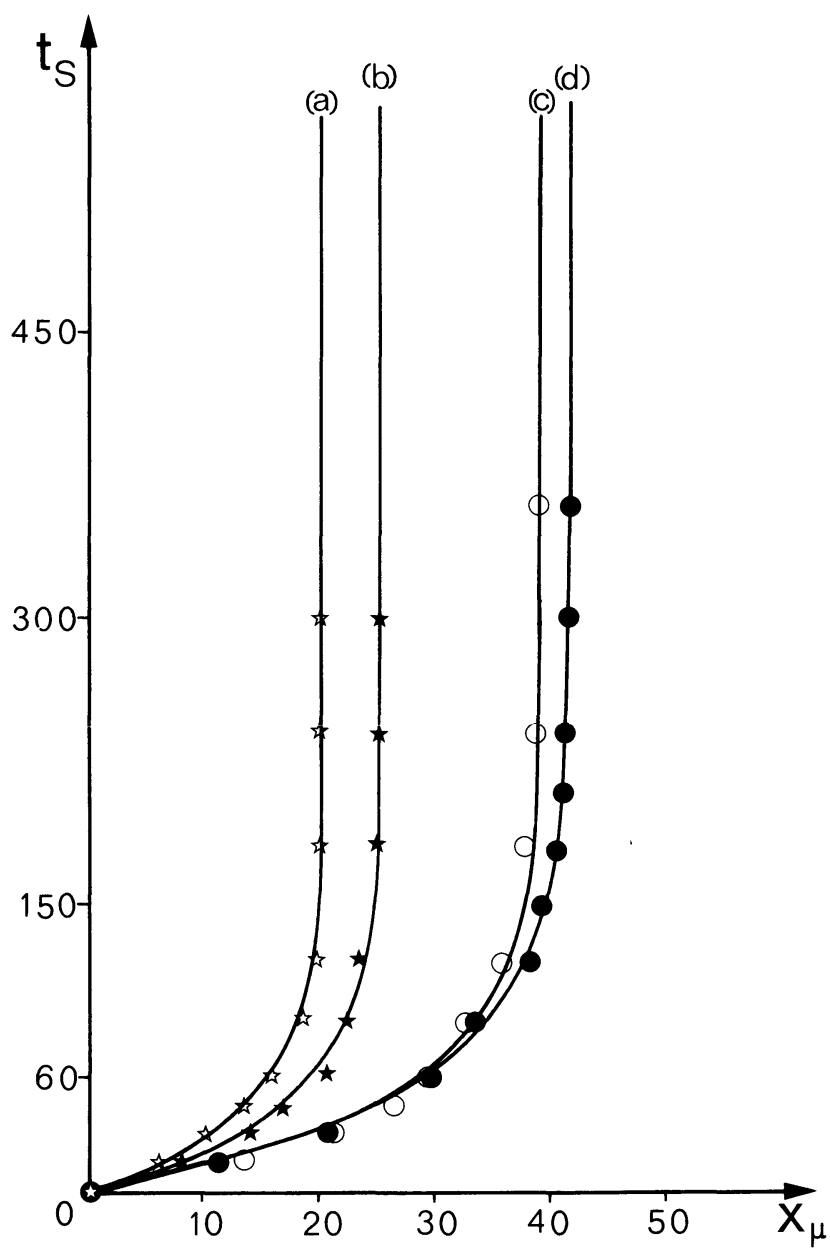

FIG. 8. - Time $t$ as a function of the line displacement $x$ in case of increasing fields and for a mixture of unperturbed pitch $P_{0}=29.7 \mu \mathrm{m}$. The full lines are theoretical, the circles and stars correspond to the experimental data. $H_{0}$ is the initial field, $H$ the applied one and $\tau_{-}$ the relaxation time.
a) $H_{0}=2960 \mathrm{G}$
$H=3940 \mathrm{G}, \quad \tau_{-}=82 \mathrm{~s}$
b) $H_{0}=0$
$H=2960 \mathrm{G}, \quad \tau_{-}=78 \mathrm{~s}$
c) $H_{0}=3020 \mathrm{G}$
$H=4840 \mathrm{G}, \quad \tau_{-}=93 \mathrm{~s}$
d) $H_{0}=2940 \mathrm{G}$
$H=4880 \mathrm{G}, \quad \tau_{-}=102 \mathrm{~s}$ 
TABLEAU I

Equilibrium position of the first single line under weak magnetic fields

\begin{tabular}{|c|c|c|c|c|c|c|}
\hline & $\begin{array}{c}\text { Unperturbed } \\
\text { pitch } \\
P_{0} \\
\mu \mathrm{m}\end{array}$ & $\begin{array}{c}\text { Applied } \\
\text { field } \\
H \\
\text { G }\end{array}$ & $\begin{array}{c}\text { Initial } \\
\text { field } \\
H_{0} \\
\mathrm{G}\end{array}$ & $\begin{array}{c}\text { Theory } \\
X_{\mathrm{e}} \\
\mu \mathrm{m}\end{array}$ & $\begin{array}{c}\text { Experiment } \\
X_{\mathrm{e}} \\
\mu \mathrm{m}\end{array}$ & $\frac{\Delta X_{\mathrm{e}}}{X_{\mathrm{e}}}$ \\
\hline & - & - & - & - & - & - \\
\hline \multirow[t]{3}{*}{ Zero field } & 29.7. & 0 & 0 & 1090 & 1045 & $4 \%$ \\
\hline & 29.9 & 0 & 0 & 1094 & 1084 & $1 \%$ \\
\hline & 33.1 & 0 & 0 & 1151 & 1125 & $2 \%$ \\
\hline \multirow[t]{7}{*}{ Increasing fields } & 29.9 & 2880 & 0 & 1117 & 1102 & $1 \%$ \\
\hline & 29.7 & 2960 & 0 & 1115 & 1064 & $5 \%$ \\
\hline & 29.7 & 3940 & 2960 & 1135 & 1084 & $5 \%$ \\
\hline & 29.7 & 4840 & 3020 & 1155 & 1098 & $5 \%$ \\
\hline & 29.7 & 4880 & 2940 & 1156 & 1105 & $4 \%$ \\
\hline & 29.9 & 6180 & 2880 & 1200 & 1189 & $1 \%$ \\
\hline & 29.7 & 6680 & 2960 & 1211 & 1192 & $2 \%$ \\
\hline \multirow[t]{3}{*}{ Decreasing fields } & 29.9 & 2920 & 6460 & 1118 & 1096 & $2 \%$ \\
\hline & 29.7 & 2940 & 3940 & 1114 & 1062 & $5 \%$ \\
\hline & 29.7 & 2960 & 4880 & 1115 & 1059 & $5 \%$ \\
\hline
\end{tabular}
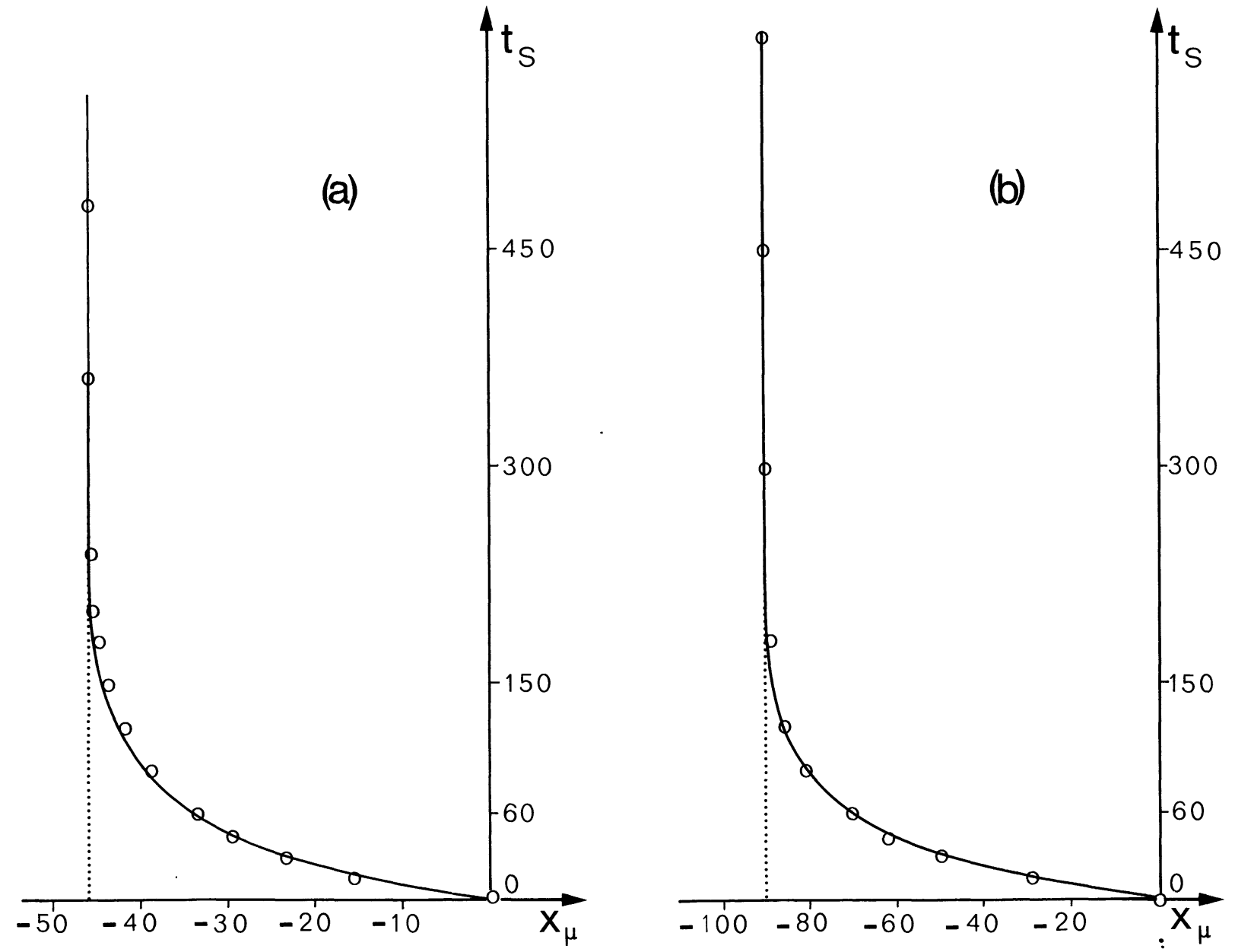

FIG. 9. - Time $t$ as a function of the line displacement $x$ in case of decreasing fields $\left(H<H_{0}\right)$. The full lines are theoretical and the open circles correspond to the experimental data.

a) $P_{0}=29.7 \mu \mathrm{m}, \quad H_{0}=5100 \mathrm{G}, \quad H=3020 \mathrm{G}, \quad \tau_{-}=83 \mathrm{~s}$ b) $P_{0}=29.9 \mu \mathrm{m}, H_{0}=6460 \mathrm{G}, H=2920 \mathrm{G}, \tau_{-}=74 \mathrm{~s}$. 
For that, it is useful to perform, in the expression for $f_{ \pm}\left(H / H_{1}\right)$ a series expansion of $\alpha$ in $H / H_{1}$. One gets :

$$
\begin{aligned}
& \tau_{-} \simeq \frac{\tau_{0}}{\sqrt{2}}\left(1+\frac{5}{8} \frac{H^{2}}{H_{1}^{2}}\right) \\
& \tau_{+} \simeq \tau_{0} \sqrt{2} \frac{H_{1}}{H}\left(1+\frac{H^{2}}{2 H_{1}^{2}}\right) .
\end{aligned}
$$

In the expression for $\tau_{-}$, the magnetic field occurs only through a second order correction term. Thus for low fields, $\tau_{-}$appears to be essentially an elastic relaxation time.

On the other hand, $\tau_{+}$diverges for low fields as $H^{-1}$. It thus appears as a magnetic relaxation time, bound to a magnetic relaxation process which disappears at zero-field.

The pure elastic relaxation time $\tau_{0}$ is obtained by plotting the theoretical curves giving $t / \tau_{0}$ versus $x$, for different values of $H_{0}$ and $H$, and fitting these curves with the experimental ones. One thus obtains :

$$
\tau_{0}=95 \pm 8 \mathrm{~s} .
$$

The motion diagrams for respectively increasing and decreasing fields are shown on figures 8 and 9 .

It is worth pointing out here that the relaxation time $\tau_{-}$is about $10^{3}$ higher than the relaxation time corresponding to the director adjustment [10]. This gives an a posteriori justification for our approach based on the separation of the dynamical study in two parts : fast reorientation of the director and then line motion.

The effective viscosity is obtained from eq. (5.2) one gets :

$$
\gamma_{\text {eff }}=450 \pm 40 \text { poises }
$$

This large value of $\gamma_{\text {eff }}$ is quite typical of line relaxation processes and explains the large values found for the relaxation times.

Finally, making use of eqs. (5.3) and (4.13), one can deduce the core radius $\varepsilon$ of the line. For that we need the twist viscous coefficient $\gamma_{1}$. It was measured for us by the Bordeaux group $\left({ }^{3}\right)$, and they found $\gamma_{1} \simeq 2.2$ poises. The ratio $K_{2} / K$ can be taken reasonably equal to 1 : then for an average local thickness $d \simeq 8 \mu \mathrm{m}$, one finds :

$$
\varepsilon=5400 \pm 500 \AA .
$$

Contrary to prevalent ideas on the size of the core line, this result suggests that core has a macroscopic size. This result is in good agreement with the one found by Geurst et al. [8] working on twisted nematics.

6. Conclusion. - A direct analysis of the magnetic effects would have been quite untractable without the rather crude assumptions we have made.

( $\left.{ }^{3}\right)$ Achard, M. F., Gasparoux, H., private communication.
The first assumption was to distinguish a fast director adjustment motion and a much slower motion of lines, and to treat them separately. The orders of magnitude of the time constant characterizing these two processes have $a$ posteriori justified this assumption.

The second assumption concerned the perturbed region around the line. We have neglected i) the thickness variation along the perturbation range, ii) the magnetic effects, as much smaller than the elastic ones in this region of strong distortion, and iii) the difference $K_{1}-K_{3}$ between the splay and bend Franck constants.

Our third crude assumption was that the core radius was proportional to the local thickness, i.e. to the distortion range around the line.

In spite of the crudeness of these assumptions, our theoretical predictions seem to account fairly well for the experimental results. This is perhaps not very surprising. Most of our assumptions have been justified above. Perhaps the least justified assumption is to take $K_{1}=K_{3}$. In fact, if $K_{1} \neq K_{3}$ one would find, as a consequence, a slight modification of the director configuration around the disclination, and hence of $(\partial \varphi / \partial x)^{2}$. This term plays a central role in the dissipation integral $V(\varepsilon / d)$. From the experimental data, we had an evaluation of $d$, hence of $\varepsilon$, the core radius. As $V$ depends of $\varepsilon / d$ through a logarithm, the slight difference in $V(\varepsilon / d)$ obtained for $K_{1} \neq K_{3}$, could have a dramatic influence on the value of $\varepsilon / d$. In other words, the macroscopic character of the core radius could effectively be a consequence of the assumption $K_{1}=K_{3}$.

The same criticism obviously applies to the analysis made by the Phillips group on disclination dynamics in twisted nematics.

The use of a computer would probably allow for a complete treatment of the general case $\left(K_{1} \neq K_{3}\right)$ and give a more reliable evaluation for the core radius.

On the other hand, one could expect the core-radius to depend on the line velocity. This would probably be true for much higher velocities than the ones achieved in these experiments. If this was true, our analysis, which takes $V$ as time-independent, would be completely wrong, since the experimental data show. strong variations of the line velocity. The agreement obtained shows that for such slow motions, one can neglect the velocity-dependence of $\varepsilon$.

We can conclude that in spite of the fact that the macroscopic character of $\varepsilon / d$ is not very strongly established, our analysis gives a good description of the dynamical process, and that the line motion is characterized by a long relaxation time $\left(\tau \simeq 10^{2} \mathrm{~s}\right)$ due to a strong effective viscosity ( $\gamma_{\text {eff }} \simeq 450$ poises). It is worth to point out that this strong viscosity is bound to a twist reorientation motion which, in a cholesteric coarse grain model, is strictly similar to permeation in smectics. Of course a coarse grain model would not apply very well for this case where 
the sample thickness is of the same order than the pitch. It remains that even in this case a calculation based on Leslie's equations predicts a very strong effective viscosity characteristic of permeation processes.

Acknowledgments. - It is a pleasure to thank here Drs. M. F. Achard, G. Sigaud and H. Gasparoux who have kindly measured for us the magnetic anisotropy susceptibility, $\chi_{a}$, and the twist viscous coefficient $\gamma_{1}$.

The magnetic measurements were made in the Centre d'Electronique des Solides (Montpellier). We are greatly indebted to Dr. A. Raymond and Pr. J. L. Robert for their help and hospitality.

The treatment of glasses with thin evaporated film of $\mathrm{SiO}$ was made with the help of Drs. J. C. Martin and G. Sagnes. Dr. M. Brunet initiated two of us in to optical experiments on Cano wedges. We wish them to find here the expression of our gratitude.

\section{APPENDIX A}

Tilt angle $\varphi(x, z)$ of the molecules for the first single line. - The twist angle $\varphi(x, z)$ between the director and $x$ direction must verify the equation (section 3.1.2)

$$
\Delta \varphi=0
$$

with the following boundary conditions :

$$
\begin{aligned}
& \varphi=0 \quad \text { at } \quad z=0 \text { and } \quad z= \pm d / 2 \quad \text { when } x<0 \\
& \varphi=0 \quad \text { at } \quad z=-d / 2 \\
& \varphi=\pi / 2 \text { at } z=0 \\
& \varphi=\pi \quad \text { at } \quad z=d / 2
\end{aligned}
$$

Therefore we must introduce a cut-off at $x=0$ when $z \geqslant 0$ (Fig. 10).

The easiest way to solve Laplace's equation is the conformal mapping method. Dęfine $\zeta=x+i z$ and :

$$
\Phi(\zeta)=\chi(x, z)+i \varphi(x, z)
$$

where $\chi$ and $\varphi$ are real functions of $x$ and $z$. If $\Phi$ is an holomorphic function in the complex plane satisfying

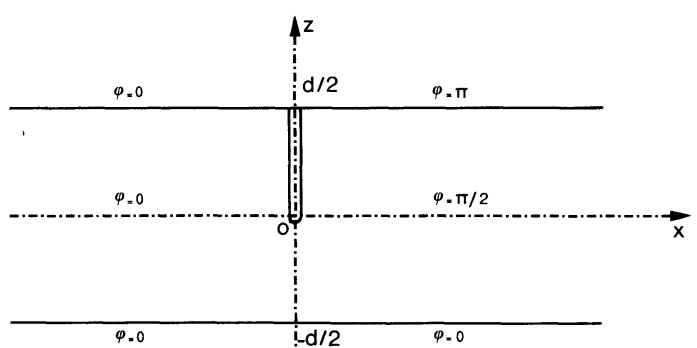

FIG. 10. - The $\zeta$-complex plane. $\Phi$ is an holomorphic function of $\zeta$ in the stripe $-\frac{d}{2} \leqslant \zeta \leqslant \frac{d}{2}$ except along the cut-off line $x=0, z \geqslant 0$. the boundary conditions (3.3), then eq. (3.2) is satisfied.

Perform now the conformal transformation

$$
Z=\exp (2 \pi \zeta / d)
$$

The stripe $-d / 2 \leqslant z \leqslant d / 2$ is now transformed into the whole complex plane. The cut-off contour is now the upper half-circle centered at the origin with radius one and the half axis $\operatorname{Im} Z=0, \operatorname{Re} Z \leqslant-1$ (Fig. 11). The boundary conditions are transformed as follows :

$\operatorname{Im} \Phi=0$ for $\operatorname{Im} Z=0$ and $1<\operatorname{Re} Z$

$\operatorname{Im} \Phi=0$ for $\operatorname{Im} Z=0$ and $-1<\operatorname{Re} Z<1$

$\operatorname{Im} \Phi=0$ for $\operatorname{Im} Z=0^{-}$and $\operatorname{Re} Z<-1$

$\operatorname{Im} \Phi=\pi$ for $\operatorname{Im} Z=0^{+}$and $\operatorname{Re} Z<-1$.

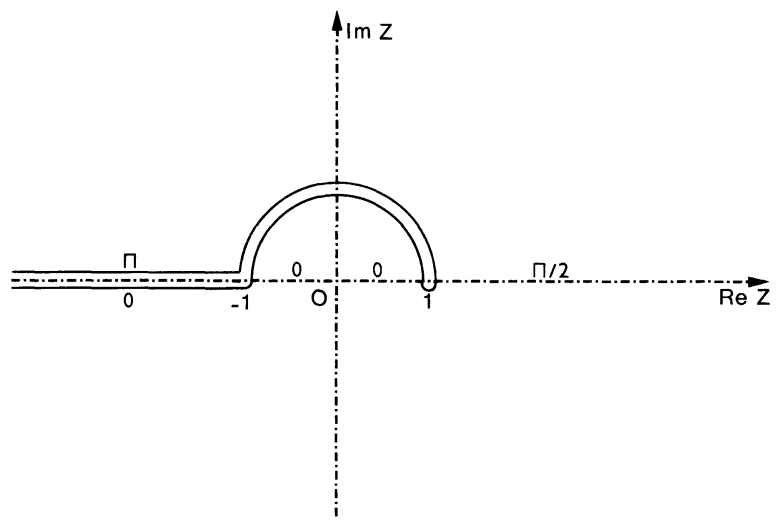

FIG. 11. - The Z-complex plane. The cut-off line is now composed of the half axis $\operatorname{Im} Z=0, \operatorname{Re} Z \leqslant-1$ and the upper half unit circle. The numbers are the numerical values of $\operatorname{Im} \Phi$ corresponding to the boundary conditions.

The $\pi$ discontinuity along the cut-off suggests the solution

$$
\Phi(Z)=\frac{1}{2} \log (Z-1)+\frac{i \pi}{2}
$$

which is holomorphic and satisfies conditions (A.3).

Hence, from (A.2) one gets :

$\Phi(\zeta)=\frac{1}{2} \log 2+\frac{1}{2} \log \operatorname{Sinh} \frac{\pi \zeta}{d}+\frac{\pi \zeta}{2 d}+\frac{i \pi}{2}$.

Taking the imaginary parts, one gets, for $x>0$

$$
\varphi(x, z)=\frac{1}{2} \operatorname{Arctan}\left(\operatorname{coth} \frac{\pi x}{d} \tan \frac{\pi z}{d}\right)+\frac{\pi}{2}+\frac{\pi z}{2 d} .
$$

For $x=0$, this function has a $\frac{\pi}{2}$ discontinuity along the lower half unit circle. In order to remove this discontinuity, one must add $-\pi / 2 E(-x)$ where $E(x)$ 
is the Heaviside function $(E(x)=0$ for $x<0$; $E(x)=1$ for $x>0)$. One then gets :

$$
\begin{aligned}
\varphi(x, z)=\frac{\pi}{2} E(x) & +\frac{\pi z}{2 d}+ \\
+ & \frac{1}{2} \operatorname{Arctan}\left(\operatorname{coth} \frac{\pi x}{d} \tan \frac{\pi z}{d}\right) .
\end{aligned}
$$

$\partial \varphi / \partial x$ and $\partial \varphi / \partial z$ can be easily derived :

$$
\begin{array}{r}
\frac{\partial \varphi}{\partial x}=\frac{\pi}{2} \delta(x)+\frac{\pi}{2 d} \frac{\tan \frac{\pi z}{d}\left[1-\operatorname{coth}^{2} \frac{\pi x}{d}\right]}{1+\operatorname{coth}^{2} \frac{\pi x}{d} \tan \frac{\pi z}{d}}+ \\
+\frac{\pi}{2} \delta(x) \operatorname{sgn}(z),
\end{array}
$$

where $\delta(x)$ is the Dirac's function. The last term of the r.h.s. results from the discontinuity of the function Arc $\tan (\operatorname{sgn}(z)=1$ for $x>0$ and $\operatorname{sgn}(z)=-1$ for $x<0)$.

Finally, $\partial \varphi / \partial x$ can be expressed as follows :

$$
\frac{\partial \varphi}{\partial x}=\pi \delta(x) E(z)-\frac{\pi}{2 d} \frac{\sin \frac{2 \pi z}{d}}{\cosh \frac{2 \pi x}{d}-\cos \frac{2 \pi z}{d}} .
$$

The term in $\delta(x)$ has no physical signification, and will not be taken into account : one therefore can write :

$$
\frac{\partial \varphi}{\partial x}=-\frac{\pi}{2 d} \frac{\sin \frac{2 \pi z}{d}}{\cosh \frac{2 \pi x}{d}-\cos \frac{2 \pi z}{d}} .
$$

Eq. (A.5) shows clearly that $\partial \varphi / \partial x$ has a significant variation only near the disclination line.

The derivation of $\partial \varphi / \partial z$ is straight forward. One finds :

$$
\frac{\partial \varphi}{\partial z}=\frac{\pi}{2 d}\left[1+\frac{\sinh \frac{2 \pi x}{d}}{\cosh \frac{2 \pi x}{d}-\cos \frac{2 \pi z}{d}}\right] .
$$

\section{APPENDIX B}

Energy of the first disclination line. - The planar structure of the first single Grandjean-Cano line was studied in section 3.1. This study was performed by assuming the cholesteric to be isotropic.

Consider now the cholesteric with elastic constants

$$
K_{1}=K_{3}=K \neq K_{2} \text {. }
$$

The tilt angle $\varphi(x, z)$ calculated in appendix $\mathrm{A}$ (eq. (3.4)) then becomes :

$$
\begin{aligned}
\varphi(x, z) & =\frac{\pi}{2} E(x)+\frac{\pi z}{2 d}+ \\
+ & \frac{1}{2} \operatorname{Arctan}\left[\operatorname{coth}\left(\frac{K_{2}}{K}\right)^{1 / 2} \frac{\pi x}{d} \tan \frac{\pi z}{d}\right] .
\end{aligned}
$$

This expression was obtained by assuming the local thickness $d$ to be constant along the strongly distorted domain $\mathrm{D}$ around the disclination line.

The distortion free-energy density is [30] :

$$
\begin{aligned}
f=\frac{K}{2}\left[(\operatorname{div} \mathbf{n})^{2}+(\mathbf{n} \times \operatorname{curl} \mathbf{n})^{2}\right] & + \\
& +\frac{K_{2}}{2}\left(\mathbf{n} \cdot \operatorname{curl} \mathbf{n}+q_{0}\right)^{2} .
\end{aligned}
$$

In this static configuration the director has the following components :

$$
n_{x}=\cos \varphi(x, z) \quad n_{y}=\sin \varphi(x, z) \quad n_{z}=0
$$

that leads to :

$$
f=\frac{K}{2}\left(\frac{\partial \varphi}{\partial x}\right)^{2}+\frac{K_{2}}{2}\left(q_{0}-\frac{\partial \varphi}{\partial z}\right)^{2} .
$$

The spatial derivatives $\partial \varphi / \partial x$ and $\partial \varphi / \partial z$ have been calculated in appendix A (eqs. A.5 and A.6). They can be written now :

$$
\begin{aligned}
& \frac{\partial \varphi}{\partial x}=-\left(\frac{K_{2}}{K}\right)^{1 / 2} \frac{\pi}{2 d} \frac{\sin \frac{2 \pi z}{d}}{\cosh \left(\frac{K_{2}}{K}\right)^{1 / 2} \frac{2 \pi x}{d}-\cos \frac{2 \pi z}{d}} \\
& \frac{\partial \varphi}{\partial z}=q_{0}+\frac{\pi}{2 d} \frac{\sinh \left(\frac{K_{2}}{K}\right)^{1 / 2} \frac{2 \pi x}{d}}{\cosh \left(\frac{K_{2}}{K}\right)^{1 / 2} \frac{2 \pi x}{d}-\cos \frac{2 \pi z}{d}} .
\end{aligned}
$$

More exactly we want to calculate the excess freeenergy of the strong distortion due to the disclination line, introduced in eq. (3.19).

This free-energy $F_{\mathrm{D}}$ (per unit length along y-axis) can then be expressed by :

$$
\begin{aligned}
& F_{\mathrm{D}}=\int_{-\infty}^{+\infty} \mathrm{d} x \int_{-d / 2}^{d / 2} \mathrm{~d} z {\left[\frac{K}{2}\left(\frac{\partial \varphi}{\partial x}\right)^{2}+\frac{K_{2}}{2}\left(q_{0}-\frac{\partial \varphi}{\partial z}\right)^{2}\right]-} \\
&-\frac{K_{2}}{2} \int_{-\infty}^{0} \mathrm{~d} x \int_{-d / 2}^{d / 2} q_{0}^{2} \mathrm{~d} z-\frac{K_{2}}{2} \int_{0}^{\infty} \times \\
& \times \mathrm{d} x \int_{-d / 2}^{d / 2}\left(\frac{\pi}{d}-q_{0}\right)^{2} \mathrm{~d} z \quad \text { (B.4) }
\end{aligned}
$$


where we have taken off the free-energy of nematic and cholesteric configurations on both sides of the disclination line (the two last terms in r.h.s. of eq. (B. 4)). Since $q_{0}=\pi / 2 d$ eq. (B. 4 ) can be rewritten :

$$
\begin{aligned}
F_{\mathrm{D}}=\frac{K}{2} & \int_{-\infty}^{+\infty} \mathrm{d} x \int_{-d / 2}^{d / 2}\left(\frac{\partial \varphi}{\partial x}\right)^{2} \mathrm{~d} z+ \\
& +\frac{K_{2}}{2} \int_{-\infty}^{+\infty} \mathrm{d} x \int_{-\mathrm{d} / 2}^{d / 2}\left[\left(q_{0}-\frac{\partial \varphi}{\partial z}\right)^{2}-q_{0}^{2}\right] \mathrm{d} z .
\end{aligned}
$$

Or

$$
F_{\mathbf{D}}=\frac{K}{2} V(d)+\frac{K_{2}}{2} W(d) .
$$

The first step is to calculate $V(d)$ :

$$
V(d)=\int_{-\infty}^{+\infty} \mathrm{d} x \int_{-d / 2}^{d / 2}\left(\frac{\partial \varphi}{\partial x}\right)^{2} \mathrm{~d} z
$$

Using the following notations :

$$
\theta=\frac{2 \pi z}{d}, \quad \psi=\frac{2 \pi x}{d}\left(\frac{K_{2}}{K}\right)^{1 / 2}
$$

and inserting eq. (B.2) into eq. (B.7), one finds :

$$
\begin{aligned}
V(d)=\frac{1}{16}\left(\frac{K_{2}}{K}\right)^{1 / 2} \int_{-\infty}^{+\infty} \mathrm{d} \psi & \int_{-\pi}^{\pi} \times \\
& \times \frac{\sin ^{2} \theta}{(\cosh \psi-\cos \theta)^{2}} \mathrm{~d} \theta .
\end{aligned}
$$

$V(d)$ diverges on the disclination line $(\psi=\theta=0)$. We shall therefore perform a cut-off excluding a small square around the line.

The domain of integration can now be divided into three parts :

$$
\begin{array}{r}
V(d)=\frac{1}{16}\left(\frac{K_{2}}{K}\right)^{1 / 2}\left[\int_{-\infty}^{-\rho} \mathrm{d} \psi \int_{-\pi}^{\pi} \frac{\sin ^{2} \theta}{(\cosh \psi-\cos \theta)^{2}} \mathrm{~d} \theta+\int_{\rho}^{\infty} \mathrm{d} \psi \int_{-\pi}^{\pi} \frac{\sin ^{2} \theta}{(\cosh \psi-\cos \theta)^{2}} \mathrm{~d} \theta+\right. \\
\left.+2 \int_{-\rho}^{\rho} \mathrm{d} \psi \int_{\rho}^{\pi} \frac{\sin ^{2} \theta}{(\cosh \psi-\cos \theta)^{2}} \mathrm{~d} \theta\right]
\end{array}
$$

where $2 \rho=\frac{4 \pi \varepsilon}{d}\left(\frac{K_{2}}{K}\right)^{1 / 2}$ is the cut-off square side (Fig. 12), $\varepsilon$ being the core radius of the disclination line. This core corresponds to the locus of undetermined orientation of the director; taking into account the smallness of the core, the choice of such a cut-off square can be considered as a good approximation. $\mathrm{S}_{3}$ is the area between the straight lines $\Psi= \pm \rho$ except for the cut-off square.

Define the following quantities

$$
V_{2}^{\prime}=\int_{-\pi}^{\pi} \frac{\sin ^{2} \theta}{(\cosh \Psi-\cos \theta)^{2}} \mathrm{~d} \theta ; \quad V_{2}=\int_{\rho}^{\infty} V_{2}^{\prime} \mathrm{d} \Psi
$$

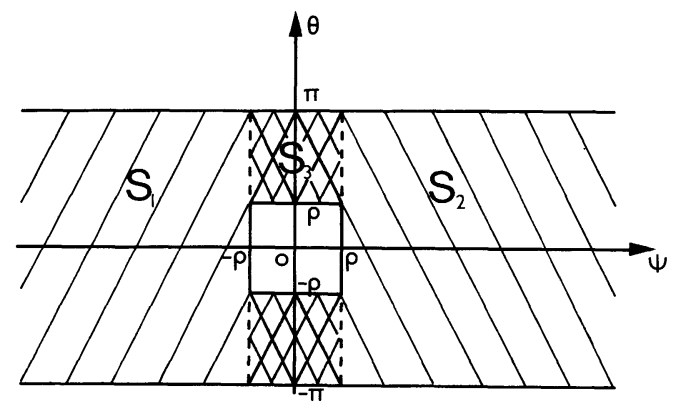

FIG. 12. - The domain of integration in the $\theta, \psi$ space is divided into three parts, $S_{1}, S_{2}$ and $S_{3}$. The cut-off region is approximated by a square whose sides are equal to $2 \rho$.

$$
V_{3}^{\prime}=\int_{\rho}^{\pi} \frac{\sin ^{2} \theta}{(\cosh \Psi-\cos \theta)^{2}} \mathrm{~d} \theta ; \quad V_{3}=2 \int_{-\rho}^{\rho} V_{3}^{\prime} \mathrm{d} \Psi
$$

Then $V$ is rewritten

$$
V=\frac{1}{16}\left(\frac{K_{2}}{K}\right)^{1 / 2}\left(2 V_{2}+V_{3}\right)
$$

We shall now perform the integrations occurring on the r.h.s. of eqs. (B.10) and (B.11). After a partial integration, one gets from eq. (B.10) :

$$
V_{2}^{\prime}=\int_{-\pi}^{\pi} \frac{\cos \theta \mathrm{d} \theta}{\cosh \psi-\cos \theta}
$$

Define now

$$
Z=\mathrm{e}^{i \theta} .
$$

Then

$$
V_{2}^{\prime}=\frac{1}{i} \int_{(\mathrm{C})} \frac{\left(z^{2}+1\right) \mathrm{d} z}{z\left(-z^{2}+2 z \cosh \psi-1\right)}
$$

where $\mathrm{C}$ is the circle with center 0 and radius 1 in the complex plane. $V_{2}^{\prime}$ is now easily integrated by residues and one gets :

$$
V_{2}^{\prime}=2 \pi(\operatorname{coth} \psi-1) .
$$


The integration on $\psi$ gives

$$
V_{2}=2 \pi\left(\ln \frac{1}{2}-\ln \frac{1-\mathrm{e}^{-2 \rho}}{2}\right) .
$$

Since $\rho \ll 1, V_{2}$ can be rewritten as

$$
V_{2} \simeq 2 \pi \ln \frac{1}{2 \rho}
$$

From eq. (B. 11), a partial integration gives

$$
V_{3}^{\prime}=\frac{\sin \rho}{\cosh \psi-\cos \rho}+(\rho-\pi)+V_{3}^{\prime \prime}
$$

where

$$
V_{3}^{\prime \prime}=\int_{\rho}^{\pi} \frac{\cosh \psi \mathrm{d} \theta}{\cosh \psi-\cos \theta}
$$

This last integral is classical. The integration gives

$$
V_{3}^{\prime \prime}=2 \operatorname{coth} \psi \operatorname{Arctan} \frac{\tanh \frac{\psi}{2}}{\tan \frac{\rho}{2}} .
$$

Since $\rho$ and $\psi$ are much smaller than one, $V_{3}^{\prime \prime}$ can be rewritten

$$
V_{3}^{\prime \prime}=\frac{2}{\psi} \operatorname{Arctan} \frac{\psi}{\rho} .
$$

From eqs. (B.11, 14 and 15) one now gets

$$
\begin{aligned}
V_{3}= & 2 \int_{-\rho}^{\rho} \frac{\sin \rho}{\cosh \psi-\cos \rho} \mathrm{d} \psi+ \\
& +2 \int_{-\rho}^{\rho}\left(\rho-\pi+\frac{2}{\psi} \operatorname{Arctan} \frac{\psi}{\rho}\right) \mathrm{d} \psi .
\end{aligned}
$$

Using the following expansions :

$$
\sin \rho \simeq \rho, \quad \cosh \psi \simeq 1+\frac{\psi^{2}}{2}, \quad \cos \rho \simeq 1-\frac{\rho^{2}}{2}
$$

the first integral in r.h.s. of eq. (B.16) is equal to $2 \pi$. The second integral is readily calculated using the relation

$$
\int_{0}^{1} \frac{\operatorname{Arctan} u}{u} \mathrm{~d} u=G
$$

where $G$ is the Catalan's constant ( $G \simeq 0.916$ ).

Then

$$
\begin{aligned}
2 \int_{-\rho}^{\rho}\left(\rho-\pi+\frac{2}{\psi} \operatorname{Arctan} \frac{\psi}{\rho}\right) \mathrm{d} \psi & = \\
& =4\left(\rho^{2}-\pi \rho+2 G\right)
\end{aligned}
$$

and finally :

$$
V_{3} \simeq 2 \pi+4\left(\rho^{2}-\pi \rho+2 G\right) .
$$

Inserting eqs. (B.13) and (B.17) into eq. (B.12), and neglecting $\rho$ and $\rho^{2}$ with respect to $\ln \frac{1}{2 \rho}$, $V$ reduces to

$$
V \simeq \frac{\pi}{4}\left(\frac{K_{2}}{K}\right)^{1 / 2}\left[\ln \frac{d}{4 \pi \varepsilon}\left(\frac{K}{K_{2}}\right)^{1 / 2}+\frac{1}{2}+\frac{2 G}{\pi}\right]
$$

The second step is to calculate $W(d)$ in eq. (B.6). From eq. (B.5) one has :

$$
W=\frac{K_{2}}{2} \int_{-\infty}^{+\infty} \mathrm{d} x \int_{-d / 2}^{d / 2}\left[\left(q_{0}-\frac{\partial \varphi}{\partial z}\right)^{2}-q_{0}^{2}\right] \mathrm{d} z
$$

Use the expression (B.3) of $\partial \varphi / \partial z$, introduce again the $\theta$ and $\psi$ variables (eq. B. 8 ), divide again the domain of integration into three parts (Fig. 12) and define the following notations :

$$
\begin{aligned}
& W_{2}^{\prime}=\int_{-\pi}^{\pi} \frac{\sinh ^{2} \psi \mathrm{d} \theta}{(\cosh \psi-\cos \theta)^{2}}-2 \pi \\
& W_{2}=\int_{\rho}^{\infty} W_{2}^{\prime} \mathrm{d} \psi \\
& W_{3}^{\prime}=\int_{\rho}^{\pi} \frac{\sinh ^{2} \psi \mathrm{d} \theta}{(\cosh \psi-\cos \theta)^{2}}-\pi \\
& W_{3}=2 \int_{-\rho}^{\rho} W_{3}^{\prime} \mathrm{d} \psi
\end{aligned}
$$

Then $W(d)$ is rewritten

$$
W=\frac{1}{16}\left(\frac{K}{K_{2}}\right)^{1 / 2}\left(2 W_{2}+W_{3}\right) .
$$

$W_{2}^{\prime}$ (eq. B. 18) is readily integrated, one obtains :

$$
W_{2}^{\prime}=2 \pi(\operatorname{coth} \psi-1) \text {. }
$$

Then recalling that $\rho \ll 1$ :

$$
W_{2} \simeq 2 \pi \ln \frac{1}{2 \rho} \text {. }
$$

Turning back to $W_{3}^{\prime}$ (eq. B. 19) one obtains :

$W_{3}^{\prime}=-\frac{\sin \rho}{\cosh \psi-\cos \rho}+$ $+\pi \operatorname{coth} \psi-2 \operatorname{coth} \psi \operatorname{Arctan}\left(\operatorname{coth} \frac{\psi}{2} \tan \frac{\rho}{2}\right)-\pi$. 
Using again the expansions of the trigonometric functions, one finds :

$$
W_{3}^{\prime} \simeq-\frac{2}{\rho} \frac{1}{1+\frac{\psi^{2}}{\rho^{2}}}+\frac{2}{\psi} \operatorname{Arctan} \frac{\psi}{\rho}-\pi
$$

then :

$$
W_{3} \simeq-4 \pi \rho-2 \pi+8 G \text {. }
$$

Insert eqs. (B.21, 22) into eq. (B. 20) and neglect the term in $\rho$ with regard to $\ln \frac{1}{2 \rho}$. Recalling that

$$
\rho=\frac{2 \pi \varepsilon}{d}\left(\frac{K_{2}}{K}\right)^{1 / 2},
$$

$W$ can be expressed in the following terms :

$$
W \simeq \frac{\pi}{4}\left(\frac{K}{K_{2}}\right)^{1 / 2}\left[\ln \frac{d}{4 \pi \varepsilon}\left(\frac{K}{K_{2}}\right)^{1 / 2}-\frac{1}{2}+\frac{2 G}{\pi}\right] .
$$

Inserting eqs. (4.13) and (B.23) into eq. (B.6) one obtains :

$$
F_{\mathrm{D}} \simeq \frac{\pi}{4}\left(K_{2} K\right)^{1 / 2}\left[\ln \frac{d}{4 \pi \varepsilon}\left(\frac{K}{K_{2}}\right)^{1 / 2}+\frac{2 G}{\pi}\right] .
$$

One remarks that in this expression the core radius $\varepsilon$ of the disclination line appears through the logarithm of $\frac{d}{\varepsilon}$. This result is quite typical of such problems.

\section{APPENDIX C}

Discussion on logarithmic terms in eq. (4.19). - In section 5.2 we have found the following motion equation :

$$
\begin{aligned}
& \frac{t^{\prime}}{V}=\frac{1}{\alpha \sqrt{\frac{\pi}{r h}}}\left\{\sqrt{1-\alpha}\left[\ln \frac{1+\frac{X^{\prime}}{2} \sqrt{\frac{h}{\pi r(1-\alpha)}}}{1-\frac{X^{\prime}}{2} \sqrt{\frac{h}{\pi r(1-\alpha)}}}-\ln \frac{1+\frac{X_{0}^{\prime}}{2} \sqrt{\frac{h}{\pi r(1-\alpha)}}}{1-\frac{X_{0}^{\prime}}{2} \sqrt{\frac{h}{\pi r(1-\alpha)}}}\right]-\right. \\
& \left.-\sqrt{1+\alpha}\left[\ln \frac{1+\frac{X^{\prime}}{2} \sqrt{\frac{h}{\pi r(1+\alpha)}}}{1-\frac{X^{\prime}}{2} \sqrt{\frac{h}{\pi r(1+\alpha)}}}-\ln \frac{1+\frac{X_{0}^{\prime}}{2} \sqrt{\frac{h}{\pi r(1+\alpha)}}}{1-\frac{X_{0}^{\prime}}{2} \sqrt{\frac{h}{\pi r(1+\alpha)}}}\right]\right\} \text {. }
\end{aligned}
$$

The equilibrium position of the line is obtained for (eq. 4.20):

$$
X_{\mathrm{e}}^{\prime}=\frac{2}{\sqrt{\frac{h}{\pi r(1-\alpha)}}}
$$

and one has :

$$
\begin{array}{llll}
X^{\prime} \leqslant X_{\mathrm{e}}^{\prime} & \text { for } \quad H>H_{0} \quad \text { (increasing fields) } \\
X^{\prime} \geqslant X_{\mathrm{e}}^{\prime} & \text { for } \quad H<H_{0} \quad \text { (decreasing fields) }
\end{array}
$$

where $H$ is the applied field and $H_{0}$ the initial one.

Therefore one can write :

$$
\frac{X^{\prime}}{2} \sqrt{\frac{h}{\pi r(1-\alpha)}}=\frac{X^{\prime}}{X_{\mathrm{e}}^{\prime}}\left\{\begin{array}{ll}
\leqslant 1 & \text { for } H>H_{0} \\
\geqslant 1 & \text { for } H<H_{0}
\end{array} .\right.
$$

This remains true for $X^{\prime}=X_{0}^{\prime}$, therefore

$$
\frac{X_{0}^{\prime}}{2} \sqrt{\frac{h}{\pi r(1-\alpha)}}=\frac{X_{0}^{\prime}}{X_{\mathrm{e}}^{\prime}}\left\{\begin{array}{ll}
<1 & \text { for } H>H_{0} \\
>1 & \text { for } H<H_{0}
\end{array} .\right.
$$

From eqs. (C.1) and (C.2) one has now :

$$
\left.\begin{array}{l}
\frac{X^{\prime}}{2} \sqrt{\frac{h}{\pi r(1+\alpha)}}<\frac{X^{\prime}}{2} \sqrt{\frac{h}{\pi r(1-\alpha)}}<1 \\
\frac{X_{0}^{\prime}}{2} \sqrt{\frac{h}{\pi r(1+\alpha)}}<\frac{X_{0}^{\prime}}{2} \sqrt{\frac{h}{\pi r(1-\alpha)}}<1
\end{array}\right\} \text { for } H>H_{0} .
$$

The case of decreasing fields $\left(H<H_{0}\right)$ for these two last terms is less obvious :

one can first write

$$
\frac{X_{0}^{\prime}}{2} \sqrt{\frac{h}{\pi r(1+\alpha)}}<\sqrt{\frac{X_{0}^{\prime 2} h}{4 \pi r}}
$$

turning back to the initial variables :

$X_{0}^{\prime}=\frac{X_{0}}{\xi}, \quad r=\frac{R}{\xi}, \quad h=\frac{1}{q_{0} \xi} \quad$ and $\quad d=\frac{X_{0}^{2}}{2 R}$

one obtains

$$
\frac{X_{0}^{\prime 2}}{2 r}=d q_{0} h
$$


moreover from eqs. (3.23) and (4.17) :

$$
\begin{gathered}
d=\frac{\pi}{2 q_{0}}\left(1+\frac{h^{2}}{8}\right) \\
h<h_{1} \text { with } h_{1}=\sqrt{2}
\end{gathered}
$$

thus

$$
d<\frac{5}{8} \frac{\pi}{q_{0}}
$$

that finally leads to :

Therefore

$$
\frac{X_{0}^{\prime}}{2} \sqrt{\frac{h}{\pi r(1+\alpha)}}<\sqrt{\frac{5}{8}}<1 .
$$$$
\frac{X^{\prime}}{2} \sqrt{\frac{h}{\pi r(1+\alpha)}}<\frac{X_{0}^{\prime}}{2} \sqrt{\frac{h}{\pi r(1+\alpha)}}<1 \text { for } H<H_{0} \text {. }
$$

One can now introduce in eq. (4.19) the functions $\tanh ^{-1}$ and coth ${ }^{-1}$. Taking into account the relations (C. 1, 2, 3, 4) one then finds :

For $H>H_{0}$ (increasing fields)

$$
\begin{aligned}
\left.\frac{t^{\prime}}{V}=\frac{2}{\alpha \sqrt{\frac{\pi}{r h}}\left\{\sqrt { 1 - \alpha } \left[\tanh ^{-1} \frac{X^{\prime}}{2}\right.\right.} \sqrt{\frac{h}{\pi r(1-\alpha)}}-\tanh ^{-1} \frac{X_{0}^{\prime}}{2} \sqrt{\frac{h}{\pi r(1-\alpha)}}\right]- \\
\left.-\sqrt{1+\alpha}\left[\tanh ^{-1} \frac{X^{\prime}}{2} \sqrt{\frac{h}{\pi r(1+\alpha)}}-\tanh ^{-1} \frac{X_{0}^{\prime}}{2} \sqrt{\frac{h}{\pi r(1+\alpha)}}\right]\right\}
\end{aligned}
$$

and, for $H<H_{0}$ (decreasing fields)

$$
\begin{aligned}
\left.\frac{t^{\prime}}{V}=\frac{2}{\alpha \sqrt{\frac{\pi}{r h}}\left\{\sqrt { 1 - \alpha } \left[\operatorname{coth}^{-1} \frac{X^{\prime}}{2}\right.\right.} \sqrt{\frac{h}{\pi r(1-\alpha)}}-\operatorname{coth}^{-1} \frac{X_{0}^{\prime}}{2} \sqrt{\frac{h}{\pi r(1-\alpha)}}\right]- \\
-\sqrt{1+\alpha}\left[\tanh ^{-1} \frac{X^{\prime}}{2} \sqrt{\frac{h}{\pi r(1+\alpha)}}-\tanh ^{-1} \frac{X_{0}^{\prime}}{2} \sqrt{\left.\left.\frac{h}{\pi r(1+\alpha)}\right]\right\} .}\right.
\end{aligned}
$$

\section{References}

[1] Frank, F. C., Discuss. Faraday Soc. 25 (1958) 1.

[2] Sackmann, E., Meiboom, S. and Snyder, L. C., J. Am. Chem. Soc. 89 (1967) 5982.

[3] De Gennes, P. G., Solid State Commun. 6 (1968) 163.

[4] Meyer, R. B., Appl. Phys. Lett. 12 (1968) 281.

[5] Durand, G., Léger, L., Rondelez, F. and Veyssie, M., Phys. Rev. Lett. 22 (1969) 227.

[6] Meyer, R. B., Appl. Phys. Eett. 14 (1969) 208.

[7] Malet, G., Marignan, J., Parodi, O., J. Physique Lett. 36 (1975) L-317.

[8] Gerritsma, C. J., Geurst, T. A. and Spruiut, A. N. J., Phys. Lett. 43A (1973) 356.

Geurst, T. A., Spruit, A. N. J. and Gerritsma, C. J., J. Physique 36 (1975) 653.

[9] Fan, C., Kramer, L., Stephen, M. J., Phys. Rev. A 2 (1970) 2482.

[10] Marignan, J., Malet, G., Parodi, O., J. Physique 37 (1976) 365.

[11] Friedel, G., Ann. Phys. 18 (1922) 487.

[12] Malthete, J., LecrercQ, M., Gabard, J., Billard, J., JaCques, J., C. R. Hebd. Séan. Acad. Sci. 273 (1971) 265.

Malthete, J., LecrercQ, M., Dvolaitzky, M., Gabard, J., Billard, J., Pontikis, V., Jacques, J., Mol. Cryst. Liq. Cryst. 23 (1973) 233.

[13] Cano, R., Chatelain, P., C. R. Hebd. Séan. Acad. Sci. 253 (1961) 1815

[14] Cano, R., Chatelain, P., C. R. Hebd. Séan. Acàd. Sci. 259 (1964) 352.

[15] Guyon, E., Pieranski, P., Boix, M., Lett. Appl. Eng. Sci. 1 (1973) 19.
Urbach, W., Guyon, E., Appl. Phys. Lett. 25 (1974) 479.

[16] Grandjean, F., C. R. Hebd. Séan. Acad. Sci. 172 (1921) 71.

[17] Friedel, G., Ann. Phys. 18 (1922) 273.

[18] Cano, R., Chatelain, P., C. R. Hebd. Séan. Acad. Sci. 253 (1961) 2081.

CANo, R., Bull. Soc. Fr. Mineral. 91 (1968) 20.

[19] Kassubeck, G. and Meier, G., Mol. Cryst. Liquid Cryst. 8 (1969) 305.

[20] Chatelain, P. and Brunet-Germain, M., C. R. Hebd. Séan. Acad. Sci. 268 (1969) 1016.

[21] ORSAY LIQUID CRYSTAL GROUP, Phys. Lett. 28A (1969) 687.

[22] De Gennes, P. G., C. R. Hebd. Séan. Acad. Sci. B 266 (1968) 571 .

[23] Kleman, M. and Friedel, J., J. Physique Colloq. 30 (1969) C 4-43.

[24] Bouligand, Y., J. Physique 35 (1974) 959.

[25] BYrd, P. F. and Fiedmann, M. D., Handbook of Elliptic Integrals (Ed. Springer, Berlin) 1971.

[26] Leslie, F. M., Mol. Cryst. Liq. Cryst. 7 (1969) 407; see also DE Gennes, P. G., The Physics of Liquid Crystals (Clarendon Press, Oxford) 1974, p. 246.

[27] Gradshteyn, I. S., RyzhiK, I. M., Table of Integrals Series and Products (Academic Press, New York and London) 1965.

[28] SiCARD, J., J. Phys. Lett. 37 (1976) 25.

[29] Morse, P. M., Feshbach, M., Methods of Theoretical Physics (Ed. Mc Graw-Hill Book Company, New York) 1953.

[30] OseEn, C. W., Trans. Faraday Soc. 29 (1933) 883.

Frank, F. C., Disc. Faraday Soc. 25 (1958) 19.

De Gennes, P. G., reference [26] p. 239. 